\title{
On genuine infinite algebraic tensor products
}

\author{
Chi-Keung Ng
}

\begin{abstract}
In this paper, we study genuine infinite tensor products of some algebraic structures. By a genuine infinite tensor product of vector spaces, we mean a vector space $\bigotimes_{i \in I} X_{i}$ whose linear maps coincide with multilinear maps on an infinite family $\left\{X_{i}\right\}_{i \in I}$ of vector spaces. After establishing its existence, we give a direct sum decomposition of $\bigotimes_{i \in I} X_{i}$ over a set $\Omega_{I ; X}$, through which we obtain a more concrete description and some properties of $\bigotimes_{i \in I} X_{i}$. If $\left\{A_{i}\right\}_{i \in I}$ is a family of unital *-algebras, we define, through a subgroup $\Omega_{I ; A}^{\mathrm{ut}} \subseteq \Omega_{I ; A}$, an interesting subalgebra $\bigotimes_{i \in I}^{\mathrm{ut}} A_{i}$. When all $A_{i}$ are $C^{*}$-algebras or group algebras, it is the linear span of the tensor products of unitary elements of $A_{i}$. Moreover, it is shown that $\bigotimes_{i \in I}^{\mathrm{ut}} \mathbb{C}$ is the group algebra of $\Omega_{I ; \mathbb{C}}^{\mathrm{ut}}$. In general, $\bigotimes_{i \in I}^{\mathrm{ut}} A_{i}$ can be identified with the algebraic crossed product of a cocycle twisted action of $\Omega_{I ; A}^{\text {ut }}$. On the other hand, if $\left\{H_{i}\right\}_{i \in I}$ is a family of inner product spaces, we define a Hilbert $C^{*}\left(\Omega_{I ; \mathbb{C}}^{\text {ut }}\right)$-module $\bar{\bigotimes}_{i \in I}^{\text {mod }} H_{i}$, which is the completion of a subspace $\bigotimes_{i \in I}^{\text {unit }} H_{i}$ of $\bigotimes_{i \in I} H_{i}$. If $\chi_{\Omega_{I ; \mathbb{C}}^{\text {ut }}}$ is the canonical tracial state on $C^{*}\left(\Omega_{I ; \mathbb{C}}^{\text {ut }}\right)$, then $\bar{\bigotimes}_{i \in I}^{\bmod } H_{i} \otimes_{\chi_{\Omega_{I} \text { ut }}} \mathbb{C}$ coincides with the Hilbert space $\bar{\bigotimes}_{i \in I}^{\phi_{1}} H_{i}$ given by a very elementary algebraic construction and is a natural dilation of the infinite direct product $\prod \otimes_{i \in I} H_{i}$ as defined by J. von Neumann. We will show that the canonical representation of $\bigotimes_{i \in I}^{\mathrm{ut}} \mathcal{L}\left(H_{i}\right)$ on $\bar{\bigotimes}_{i \in I}^{\phi_{1}} H_{i}$ is injective (note that the canonical representation of $\bigotimes_{i \in I}^{\mathrm{ut}} \mathcal{L}\left(H_{i}\right)$ on $\prod \otimes_{i \in I} H_{i}$ is not injective). We will also show that if $\left\{A_{i}\right\}_{i \in I}$ is a family of unital Hilbert algebras, then so is $\bigotimes_{i \in I}^{\mathrm{ut}} A_{i}$.
\end{abstract}

\section{Introduction}

In this paper, we study infinite tensor products of some algebraic structures. In the literature, infinite tensor products are often defined as inductive limit of finite tensor products (see, e.g., [4], [21] [9], [14], and [15]). As far as we know, the only

Mathematics Subject Classification (2010): Primary 15A69, 46M05; Secondary 16G99, 16S35, 20C07, 46C05, 46L99, 47A80.

Keywords: Infinite tensor products, unital *-algebras, twisted crossed products, inner product spaces, representations. 
alternative approach so far is the one by J. von Neumann, concerning infinite direct products of Hilbert spaces (see [20]). Some authors used this approach to define infinite tensor products of other functional analytic structures (see, e.g., [3], [11] and [13]). The work of von Neumann attracted the attention of many physicists who are interested in "quantum mechanics with infinite degrees of freedom", as well as mathematicians whose interest is in the field of operator algebras (see, e.g., [1], [2], [3], [8], [12], [17], and [19]).

However, von Neumann's approach is not appropriate for purely algebraic objects. The aim of this article is to study "genuine infinite algebraic tensor products" (i.e. ones that are defined in terms of multilinear maps instead of through inductive limits) of some algebraic structures. There are several motivations behind this study.

1. Conceptually speaking, it is natural to define "infinite tensor products" as the object that produces a unique linear map from a multilinear map on a given infinite family of objects (see Definition 2.1). As infinite direct products of Hilbert spaces are important in both physics and mathematics, it is believed that such infinite tensor products of algebraic structures are also important.

2. We want to construct an infinite tensor product of Hilbert spaces that is easier for non-analyst to grasp (compare with the infinite direct product as defined by J. von Neumann; see Lemma 4.2 and Remark $4.7(\mathrm{~d})$ ) and is more natural (see Theorem 4.8, Example 4.10 and Example 5.6).

3. Given a family of groups $\left\{G_{i}\right\}_{i \in I}$, it is well known that the group algebra of the group

$$
\bigoplus_{i \in I} G_{i}:=\left\{\left[g_{i}\right]_{i \in I} \in \Pi_{i \in I} G_{i}: g_{i}=e \text { except for finite number of } i \in I\right\}
$$

is an inductive limit of finite tensor products. However, if one wants to consider the group algebra $\mathbb{C}\left[\Pi_{i \in I} G_{i}\right]$, one is forced to consider a "bigger version of tensor products" (see Example 3.1).

In this article, the algebraic structures that we consider are vector spaces, unital *-algebras, inner product spaces, and *-representations of unital *-algebras on Hilbert spaces. In our study, we discovered some interesting phenomena about infinite tensor products that do not have counterparts in the case of finite tensor products. Most of these phenomena relate to a certain object, $\Omega_{I ; X}$, defined as in Remark 2.4(d), which "encodes the asymptotic information" of a given family $\left\{X_{i}\right\}_{i \in I}$.

In Section 2, we will begin our study by defining the infinite tensor product $\left(\bigotimes_{i \in I} X_{i}, \Theta_{X}\right)$ of a family $\left\{X_{i}\right\}_{i \in I}$ of vector spaces. Two particular concerns are bases of $\bigotimes_{i \in I} X_{i}$ and the relationship between $\bigotimes_{i \in I} X_{i}$ and inductive limits of finite tensor products of $\left\{X_{i}\right\}_{i \in I}$ (which depend on choices of fixed elements in $\prod_{i \in I} X_{i}$ ). In order to do this, we obtain a direct sum decomposition of $\bigotimes_{i \in I} X_{i}$ indexed by a set $\Omega_{I ; X}$ (see Theorem 2.5) with all the direct summands being inductive limits of finite tensor products (see Proposition 2.6(b)). From this, we also obtain that the canonical map

$$
\Psi: \bigotimes_{i \in I} L\left(X_{i} ; Y_{i}\right) \rightarrow L\left(\bigotimes_{i \in I} X_{i} ; \bigotimes_{i \in I} Y_{i}\right)
$$


is injective (but not surjective). As a consequence, $\bigotimes_{i \in I} X_{i}$ is automatically a faithful module over the big unital commutative algebra $\bigotimes_{i \in I} \mathbb{C}$ (see Corollary 2.9 and Example 2.10). Moreover, one may regard the canonical map

$$
\Theta_{\mathbb{C}}: \Pi_{i \in I} \mathbb{C} \rightarrow \bigotimes_{i \in I} \mathbb{C}
$$

as a generalized multiplication (see Example 2.10(a)). In this sense, one can make sense of infinite products like $(-1)^{I}$.

Clearly, $\bigotimes_{i \in I} A_{i}$ is a unital *-algebra if all $A_{i}$ are unital *-algebras. We will study in Section 3, a natural *-subalgebra $\bigotimes_{i \in I}^{\mathrm{ut}} A_{i}$ of $\bigotimes_{i \in I} A_{i}$ which is a direct sum over a subgroup $\Omega_{I ; A}^{\text {ut }}$ of the semigroup $\Omega_{I ; A}$. The reasons for considering this subalgebra are that it has good representations (see the discussion after Proposition 5.1), and it is big enough to contain $\mathbb{C}\left[\Pi_{i \in I} G_{i}\right]$ when $A_{i}=\mathbb{C}\left[G_{i}\right]$ for all $i \in I$ (see Example $3.1(\mathrm{a})$ ). Moreover, if all $A_{i}$ are generated by their unitary elements (in particular, if $A_{i}$ are group algebras or $C^{*}$-algebras), then $\bigotimes_{i \in I}^{\mathrm{ut}} A_{i}$ is the linear span of the tensor products of unitary elements in $A_{i}$. We will show that $\bigotimes_{i \in I}^{\text {ut }} A_{i}$ can be identified with the crossed products of some twisted actions in the sense of Busby and Smith (i.e., a cocycle action with a 2-cocycle) of $\Omega_{I ; A}^{\mathrm{ut}}$ on $\bigotimes_{i \in I}^{e} A_{i}$ (the unital *-algebra inductive limit of finite tensor products of $A_{i}$ ). Moreover, it is shown that $\bigotimes_{i \in I}^{\mathrm{ut}} \mathbb{C}$ can be identified with the group algebra of $\Omega_{I ; \mathbb{C}}^{\mathrm{ut}}$ (Corollary 3.4). We will also study the center of $\bigotimes_{i \in I}^{\mathrm{ut}} A_{i}$ in the case when $A_{i}$ is generated by its unitary elements (for all $i \in I$ ).

In Section 4, we will consider tensor products of inner product spaces. If $\left\{H_{i}\right\}_{i \in I}$ is a family of inner product spaces, we define a natural inner product on a subspace $\bigotimes_{i \in I}^{\text {unit }} H_{i}$ of $\bigotimes_{i \in I} H_{i}$ (see Lemma $4.2(\mathrm{~b})$ ). In the case of Hilbert spaces, the completion $\bar{\bigotimes}_{i \in I}^{\phi_{1}} H_{i}$ of $\bigotimes_{i \in I}^{\text {unit }} H_{i}$ is a "natural dilation" of the infinite direct product $\prod \otimes_{i \in I} H_{i}$ as defined by J. von Neumann in [20] (see Remark $4.7(\mathrm{~b})$ ). Note that the construction for $\bar{\bigotimes}_{i \in I}^{\phi_{1}} H_{i}$ is totally algebraical and is more natural (see Example 4.10 and Example 5.6). Note also that one can construct $\prod \otimes_{i \in I} H_{i}$ in a similar way as $\bar{\bigotimes}_{i \in I}^{\phi_{1}} H_{i}$ (see Remark $4.7(\mathrm{~d})$ ). On the other hand, there is an inner product $\mathbb{C}\left[\Omega_{I ; \mathbb{C}}^{\text {ut }}\right]$-module structure on $\bigotimes_{i \in I}^{\text {unit }} H_{i}$ which produces $\bar{\bigotimes}_{i \in I}^{\phi_{1}} H_{i}$ (see Theorem 4.8), as well as many other pre-inner products on $\bigotimes_{i \in I}^{\text {unit }} H_{i}$ (see Remark 4.9 (a)).

Section 5 will be devoted to the study of *-representations of unital *-algebras. More precisely, if $\Psi_{i}: A_{i} \rightarrow \mathcal{L}\left(H_{i}\right)$ is a unital ${ }^{*}$-representation $(i \in I)$, we define a canonical *-representation

$$
\bigotimes_{i \in I}^{\phi_{1}} \Psi_{i}: \bigotimes_{i \in I}^{\mathrm{ut}} A_{i} \rightarrow \mathcal{L}\left(\bar{\bigotimes}_{i \in I}^{\phi_{1}} H_{i}\right)
$$

We will show in Theorem 5.3 (c) that if all the $\Psi_{i}$ are injective, then $\bigotimes_{i \in I}^{\phi_{1}} \Psi_{i}$ is also injective. This is equivalent to the canonical *-representations of $\bigotimes_{i \in I}^{\text {ut }} \mathcal{L}\left(H_{i}\right)$ on $\bar{\bigotimes}_{i \in I}^{\phi_{1}} H_{i}$ being injective, and is related to the "strong faithfulness" of the canonical action of $\Omega_{I ; \mathcal{L}(H)}^{\mathrm{ut}}$ on $\Omega_{I ; H}^{\mathrm{unit}}$ (see Remark 5.4(b)). Note however, that the corresponding tensor type representation of $\bigotimes_{i \in I}^{\mathrm{ut}} \mathcal{L}\left(H_{i}\right)$ on $\prod \otimes_{i \in I} H_{i}$ is not injective. 
Consequently, if $\left(H_{i}, \pi_{i}\right)$ is a unitary representation of a group $G_{i}$ that induces an injective ${ }^{*}$-representation of $\mathbb{C}\left[G_{i}\right]$ on $H_{i}(i \in I)$, then we obtain an injective "tensor type" *-representation of $\mathbb{C}\left[\Pi_{i \in I} G_{i}\right]$ on $\bar{\bigotimes}_{i \in I}^{\phi_{1}} H_{i}$ (see Corollary 5.7). On the other hand, we will show that $\bigoplus_{\rho \in \Pi_{i \in I} S\left(A_{i}\right)}\left(\bar{\bigotimes}_{i \in I}^{\phi_{1}} H_{\rho_{i}}, \bigotimes_{i \in I}^{\phi_{1}} \pi_{\rho_{i}}\right)$ is an injective ${ }^{*}$-representation of $\bigotimes_{i \in I}^{\text {ut }} A_{i}$ when all the $A_{i}$ are $C^{*}$-algebras (Corollary 5.9). Finally, we show that if all the $A_{i}$ are unital Hilbert algebras, then so is $\bigotimes_{i \in I}^{\mathrm{ut}} A_{i}$.

Notation 1.1. i) In this article, all the vector spaces, algebras as well as inner product spaces are over the complex field $\mathbb{C}$, although some results remain valid if one considers the real field instead.

ii) Throughout this article, $I$ is an infinite set, and $\mathfrak{F}$ is the set of all non-empty finite subsets of $I$.

iii) For any vector space $X$, we write $X^{\times}:=X \backslash\{0\}$ and define $X^{*}$ to be the set of linear functionals on $X$. If $Y$ is another vector space, we denote by $X \otimes Y$ and $L(X ; Y)$ respectively, the algebraic tensor product of $X$ and $Y$, and the set of linear maps from $X$ to $Y$. We also write $L(X):=L(X ; X)$.

iv) If $\left\{X_{i}\right\}_{i \in I}$ is a family of vector spaces and $x \in \Pi_{i \in I} X_{i}$, we denote by $x_{i}$ the " $i$ th-coordinate" of $x$ (i.e. $x=\left[x_{i}\right]_{i \in I}$ ). If $x, y \in \prod_{i \in I} X_{i}$ are such that $x_{i}=y_{i}$ except for a finite number of $i \in I$, we write

$$
x_{i}=y_{i} \quad \text { e.f. }
$$

v) If $V$ is a normed space, we denote by $\mathcal{L}(V)$ and $V^{\prime}$ the set of bounded linear operators and the set of bounded linear functionals, respectively, on $V$. Moreover, we set $\mathfrak{S}_{1}(V):=\{x \in V:\|x\|=1\}$ and $B_{1}(V):=\{x \in V:\|x\| \leq 1\}$.

vi) If $A$ is a unital *-algebra, we denote by $e_{A}$ the identity of $A$ and write $U_{A}:=\left\{a \in A: a^{*} a=e_{A}=a a^{*}\right\}$.

\section{Tensor products of vector spaces}

In this section, $\left\{X_{i}\right\}_{i \in I}$ and $\left\{Y_{i}\right\}_{i \in I}$ are families of non-zero vector spaces.

Definition 2.1. Let $Y$ be a vector space. A map $\Phi: \Pi_{i \in I} X_{i} \rightarrow Y$ is said to be multilinear if $\Phi$ is linear on each variable. Suppose that $\bigotimes_{i \in I} X_{i}$ is a vector space and $\Theta_{X}: \Pi_{i \in I} X_{i} \rightarrow \bigotimes_{i \in I} X_{i}$ is a multilinear map such that for any vector space $Y$ and any multilinear map $\Phi: \Pi_{i \in I} X_{i} \rightarrow Y$, there exists a unique linear map $\tilde{\Phi}: \bigotimes_{i \in I} X_{i} \rightarrow Y$ with $\Phi=\tilde{\Phi} \circ \Theta_{X}$. Then $\left(\bigotimes_{i \in I} X_{i}, \Theta_{X}\right)$ is called the tensor product of $\left\{X_{i}\right\}_{i \in I}$. We will denote $\otimes_{i \in I} x_{i}:=\Theta_{X}(x)\left(x \in \Pi_{i \in I} X_{i}\right)$ and set $X^{\otimes I}:=\bigotimes_{i \in I} X_{i}$ if all $X_{i}$ are equal to the same vector space $X$.

Let us first give the following simple example showing that non trivial multilinear maps with an infinite number of variables do exist. They are also crucial for some constructions later on. 
Example 2.2. (a) Let $\Pi_{i \in I}^{1} \mathbb{C}:=\left\{\beta \in \Pi_{i \in I} \mathbb{C}: \beta_{i}=1\right.$ e.f. $\}$ and set

$$
\varphi_{1}(\beta):= \begin{cases}\Pi_{i \in I} \beta_{i} & \text { if } \beta \in \Pi_{i \in I}^{1} \mathbb{C} \\ 0 & \text { otherwise. }\end{cases}
$$

It is not hard to check that $\varphi_{1}$ is a non-zero multilinear map from $\Pi_{i \in I} \mathbb{C}$ to $\mathbb{C}$. If $\phi_{1}: \bigotimes_{i \in I} \mathbb{C} \rightarrow \mathbb{C}$ is the linear functional induced by $\varphi_{1}$ (the existence of $\bigotimes_{i \in I} \mathbb{C}$ will be established in Proposition $2.3(\mathrm{a})$ ), then $\phi_{1}$ is an involutive unital map.

(b) Let $\Pi_{i \in I}^{0} \mathbb{C}:=\left\{\beta \in \Pi_{i \in I} \mathbb{C}: \sum_{i \in I}\left|\beta_{i}-1\right|<\infty\right\}$. For each $\beta \in \Pi_{i \in I}^{0} \mathbb{C}$, the net $\left\{\Pi_{i \in F} \beta_{i}\right\}_{F \in \mathfrak{F}}$ converges to a complex number, denoted by $\Pi_{i \in I} \beta_{i}$ (see, e.g., 2.4.1 in [20]). We define $\varphi_{0}(\beta):=\Pi_{i \in I} \beta_{i}$ whenever $\beta \in \Pi_{i \in I}^{0} \mathbb{C}$ and set $\left.\varphi_{0}\right|_{\Pi_{i \in I} \mathbb{C} \backslash \Pi_{i \in I}^{0} \mathbb{C}} \equiv 0$. As in part (a), $\varphi_{0}$ induces an involutive unital linear functional $\phi_{0}$ on $\bigotimes_{i \in I} \mathbb{C}$.

Clearly, infinite tensor products are unique (up to linear bijections) if they exist. The existence of infinite tensor products follows from a similar argument as that for finite tensor products, but we give an outline here for future reference.

Proposition 2.3. (a) The tensor product $\left(\bigotimes_{i \in I} X_{i}, \Theta_{X}\right)$ exists.

(b) If $\left\{A_{i}\right\}_{i \in I}$ is a family of algebras (respectively, ${ }^{*}$-algebras), then $\bigotimes_{i \in I} A_{i}$ is an algebra (respectively, $a^{*}$-algebra) with $\left(\otimes_{i \in I} a_{i}\right)\left(\otimes_{i \in I} b_{i}\right):=\otimes_{i \in I} a_{i} b_{i}$ (and $\left.\left(\otimes_{i \in I} a_{i}\right)^{*}:=\left(\otimes_{i \in I} a_{i}^{*}\right)\right)$ for $a, b \in \prod_{i \in I} A_{i}$.

(c) If $\Psi_{i}: A_{i} \rightarrow L\left(X_{i}\right)$ is a homomorphism for each $i \in I$, there is a canonical homomorphism $\tilde{\bigotimes}_{i \in I} \Psi_{i}: \bigotimes_{i \in I} A_{i} \rightarrow L\left(\bigotimes_{i \in I} X_{i}\right)$ such that

$$
\left(\tilde{\bigotimes}_{i \in I} \Psi_{i}\right)\left(\otimes_{i \in I} a_{i}\right) \otimes_{i \in I} x_{i}=\otimes_{i \in I} \Psi_{i}\left(a_{i}\right) x_{i} \quad\left(a \in \Pi_{i \in I} A_{i} ; x \in \Pi_{i \in I} X_{i}\right) .
$$

(d) If $A=\bigoplus_{n=0}^{\infty} A_{n}$ is a graded algebra and $\bigoplus_{n=0}^{\infty} M_{n}$ is a graded left $A$ module, then $\bigoplus_{n=0}^{\infty} \bigotimes_{k \geq n} M_{k}$ is a graded $A$-module with

$$
a_{m}\left(\otimes_{k \geq n} x_{k}\right)=\otimes_{k \geq n} a_{m} x_{k} \in \bigotimes_{k \geq m+n} M_{k}\left(a_{m} \in A_{m} ; x \in \Pi_{k \geq n} M_{k}\right) .
$$

Proof. Parts (b), (c) and (d) follow from the universal property of tensor products, and we will only give a brief account for part (a). Let $V$ be the free vector space generated by elements in $\Pi_{i \in I} X_{i}$ and let $\Theta_{0}: \Pi_{i \in I} X_{i} \rightarrow V$ be the canonical map. Suppose that $W:=\operatorname{span} W_{0}$, where

$$
W_{0}:=\left\{\lambda \Theta_{0}(u)+\Theta_{0}(v)-\Theta_{0}(w): \lambda \in \mathbb{C} ; u, v, w \in \Pi_{i \in I} X_{i} ; \exists i_{0} \in I\right. \text { with }
$$

$$
\left.\lambda u_{i_{0}}+v_{i_{0}}=w_{i_{0}} \text { and } u_{j}=v_{j}=w_{j}, \forall j \in I \backslash\left\{i_{0}\right\}\right\} .
$$

If we put $\bigotimes_{i \in I} X_{i}:=V / W$, and set $\Theta_{X}$ to be the composition of $\Theta_{0}$ with the quotient map from $V$ to $V / W$, then they will satisfy the requirement in Definition 2.1. 
In the following remark, we list some observations that will be used implicitly throughout this article.

Remark 2.4. (a) As $\Theta_{X}$ is multilinear, $\bigotimes_{i \in I} X_{i}=\operatorname{span} \Theta_{X}\left(\Pi_{i \in I} X_{i}^{\times}\right)$.

(b) If $I_{1}$ and $I_{2}$ are non-empty disjoint subsets of $I$ with $I=I_{1} \cup I_{2}$, it follows, from the universal property, that $\bigotimes_{i \in I} X_{i} \cong\left(\bigotimes_{i \in I_{1}} X_{i}\right) \otimes\left(\bigotimes_{j \in I_{2}} X_{j}\right)$ canonically.

(c) $\bigotimes_{i \in I}\left(X_{i} \otimes Y_{i}\right) \cong\left(\bigotimes_{i \in I} X_{i}\right) \otimes\left(\bigotimes_{i \in I} Y_{i}\right)$ canonically.

(d) For any $x, y \in \Pi_{i \in I} X_{i}^{\times}$, we write

$$
x \sim y \quad \text { if } \quad x_{i}=y_{i} \text { e.f. }
$$

Obviously, $\sim$ is an equivalence relation on $\Pi_{i \in I} X_{i}^{\times}$, and we set $[x]_{\sim}$ to be the equivalence class of $x \in \Pi_{i \in I} X_{i}^{\times}$. Let $\Omega_{I ; X}$ be the collection of such equivalence classes. It is not hard to see that $\Omega_{I ; \mathbb{C}}$ is a quotient group of $\Pi_{i \in I} \mathbb{C}^{\times}$, and that it acts freely on $\Omega_{I ; X}$.

(e) The element $\otimes_{i \in I} 1 \in \mathbb{C}^{\otimes I}$ is non-zero. In fact, if $\otimes_{i \in I} 1=0$, then $\mathbb{C}^{\otimes I}=(0)$ (by Proposition $2.3(\mathrm{~b})$ ), and this implies the only multilinear map from $\Pi_{i \in I} \mathbb{C}$ to $\mathbb{C}$ is zero, which contradicts Example 2.2.

The "asymptotic object" $\Omega_{I ; X}$ defined in (d) above is crucial in the study of genuine infinite tensor products, as can be seen from our next result. Let us first give some more notation here. For every $u \in \Pi_{i \in I} X_{i}^{\times}$, we set

$$
\Pi_{i \in I}^{u} X_{i}:=\left\{x \in \Pi_{i \in I} X_{i}: x \sim u\right\} \quad \text { and } \bigotimes_{i \in I}^{u} X_{i}:=\operatorname{span} \Theta_{X}\left(\Pi_{i \in I}^{u} X_{i}\right) .
$$

If $u \sim v$, then $\Pi_{i \in I}^{u} X_{i}=\Pi_{i \in I}^{v} X_{i}$, and we will also write $\Pi_{i \in I}^{[u]} X_{i}:=\Pi_{i \in I}^{u} X_{i}$ and $\bigotimes_{i \in I}^{[u]_{\sim}} X_{i}:=\bigotimes_{i \in I}^{u} X_{i}$.

Theorem 2.5. $\bigotimes_{i \in I} X_{i}=\bigoplus_{\omega \in \Omega_{I ; X}} \bigotimes_{i \in I}^{\omega} X_{i}$.

Proof. Suppose that $x^{(1)}, \ldots, x^{(n)} \in \Pi_{i \in I} X_{i}^{\times}$and that $0=n_{0}<\cdots<n_{N}=n$ is a sequence satisfying $x^{\left(n_{k}+1\right)} \sim \cdots \sim x^{\left(n_{k+1}\right)}$ for $k \in\{0, \ldots, N-1\}$, but $x^{\left(n_{k}\right)} \nsim x^{\left(n_{l}\right)}$ whenever $1 \leq k \neq l \leq N$. We first show that if $\nu_{1}, \ldots, \nu_{n} \in \mathbb{C}$ with $\sum_{l=1}^{n} \nu_{l} \Theta_{X}\left(x^{(l)}\right)=0$, then

$$
\sum_{l=n_{k}+1}^{n_{k+1}} \nu_{l} \Theta_{X}\left(x^{(l)}\right)=0 \quad(k=0, \ldots, N-1) .
$$

In fact, by the proof of Proposition $2.3(\mathrm{a})$, there exist $m \in \mathbb{N}, \mu_{1}, \ldots, \mu_{m} \in \mathbb{C}$ and $\lambda_{k} \Theta_{0}\left(u^{(k)}\right)+\Theta_{0}\left(v^{(k)}\right)-\Theta_{0}\left(w^{(k)}\right) \in W_{0}(k=1, \ldots, m)$ such that

$$
\sum_{l=1}^{n} \nu_{l} \Theta_{0}\left(x^{(l)}\right)=\sum_{k=1}^{m} \mu_{k}\left(\lambda_{k} \Theta_{0}\left(u^{(k)}\right)+\Theta_{0}\left(v^{(k)}\right)-\Theta_{0}\left(w^{(k)}\right)\right) .
$$

Observe that if one of the elements in $\left\{u^{(k)}, v^{(k)}, w^{(k)}\right\}$ is equivalent to $x^{(1)}$ (under $\sim$ ), then so are the other two (see (2.1)). After renaming, one may assume that $u^{(k)} \sim v^{(k)} \sim w^{(k)} \sim x^{(1)}$ for $k=1, \ldots, m_{1}$, but none of $u^{(k)}, v^{(k)}$ and $w^{(k)}$ is equivalent to $x^{(1)}$ when $k \in\left\{m_{1}+1, \ldots, m\right\}$. 
Since the two sets

$\left\{x^{\left(n_{1}+1\right)}, \ldots, x^{(n)}\right\} \cup\left\{u^{\left(m_{1}+1\right)}, \ldots, u^{(m)}\right\} \cup\left\{v^{\left(m_{1}+1\right)}, \ldots, v^{(m)}\right\} \cup\left\{w^{\left(m_{1}+1\right)}, \ldots, w^{(m)}\right\}$

and

$$
\left\{x^{(1)}, \ldots, x^{\left(n_{1}\right)}\right\} \cup\left\{u^{(1)}, \ldots, u^{\left(m_{1}\right)}\right\} \cup\left\{v^{(1)}, \ldots, v^{\left(m_{1}\right)}\right\} \cup\left\{w^{(1)}, \ldots, w^{\left(m_{1}\right)}\right\}
$$

are disjoint and elements in $\Theta_{0}\left(\Pi_{i \in I} X_{i}\right)$ are linearly independent in $V$, we have

$$
\sum_{l=1}^{n_{1}} \nu_{l} \Theta_{0}\left(x^{(l)}\right)-\sum_{k=1}^{m_{1}} \mu_{k}\left(\lambda_{k} \Theta_{0}\left(u^{(k)}\right)+\Theta_{0}\left(v^{(k)}\right)-\Theta_{0}\left(w^{(k)}\right)\right)=0 .
$$

This implies that $\sum_{l=1}^{n_{1}} \nu_{l} \Theta_{X}\left(x^{(l)}\right)=0$. Similarly, $\sum_{l=n_{k}+1}^{n_{k+1}} \nu_{l} \Theta_{X}\left(x^{(l)}\right)=0$ for $k=1, \ldots, N-1$.

The above shows that

$$
\left(\bigotimes_{i \in I}^{\omega_{M}} X_{i}\right) \cap\left(\sum_{k=1}^{M-1} \bigotimes_{i \in I}^{\omega_{k}} X_{i}\right)=\{0\}
$$

when $\omega_{1}, \ldots, \omega_{M}$ are distinct elements in $\Omega_{I ; X}$. On the other hand, for every $x \in \Pi_{i \in I} X_{i}^{\times}$, one has $\Theta_{X}(x) \in \bigotimes_{i \in I}^{[x]} X_{i}$. These give the required equality.

For any $F \in \mathfrak{F}$ and $u \in \Pi_{i \in I} X_{i}^{\times}$, one has a linear map

$$
J_{F}^{u}: \bigotimes_{i \in F} X_{i} \longrightarrow \bigotimes_{i \in I}^{u} X_{i}
$$

given by $J_{F}^{u}\left(\otimes_{i \in F} x_{i}\right):=\otimes_{j \in I} \tilde{x}_{j}\left(x_{i} \in X_{i}\right)$, where $\tilde{x}_{j}:=x_{j}$ when $j \in F$, and $\tilde{x}_{j}:=u_{j}$ when $j \in I \backslash F$.

For any $F, G \in \mathfrak{F}$ with $F \subseteq G$, a similar construction gives a linear map $J_{G ; F}^{u}$ : $\bigotimes_{i \in F} X_{i} \rightarrow \bigotimes_{i \in G} X_{i}$. It is clear that $\left(\bigotimes_{i \in F} X_{i}, J_{G ; F}^{u}\right)_{F \subseteq G \in \mathfrak{F}}$ is an inductive system in the category of vector spaces with linear maps as morphisms.

Proposition 2.6. (a) $J_{F}^{u}$ is injective for any $u \in \Pi_{i \in I} X_{i}^{\times}$and $F \in \mathfrak{F}$. Consequently, $\Theta_{X}(u) \neq 0$.

(b) The inductive limit of $\left(\bigotimes_{i \in F} X_{i}, J_{G ; F}^{u}\right)_{F \subseteq G \in \mathfrak{F}}$ is $\left(\bigotimes_{i \in I}^{u} X_{i},\left\{J_{F}^{u}\right\}_{F \in \mathfrak{F}}\right)$.

Proof. (a) Suppose that $a \in \operatorname{ker} J_{F}^{u}$ and $\psi \in\left(\bigotimes_{i \in F} X_{i}\right)^{*}$. For each $j \in I \backslash F$, choose $f_{j} \in X_{j}^{*}$ with $f_{j}\left(u_{j}\right)=1$. Remark $2.4(\mathrm{~b})$ and the universal property give a linear map $\check{\psi}: \bigotimes_{i \in I} X_{i} \rightarrow \mathbb{C}^{\otimes I}$ satisfying

$$
\check{\psi}\left(\otimes_{i \in I} x_{i}\right)=\psi\left(\otimes_{i \in F} x_{i}\right)\left(\otimes_{j \in I \backslash F} f_{j}\left(x_{j}\right)\right) \quad\left(x \in \Pi_{i \in I} X_{i}\right) .
$$

Thus, $\psi(a)\left(\otimes_{i \in I} 1\right)=\check{\psi}\left(J_{F}^{u}(a)\right)=0$, which implies that $a=0$ (as $\psi$ is arbitrary) as required. On the other hand, if $i_{0} \in I$, then $\Theta_{X}(u)=J_{\left\{i_{0}\right\}}^{u}\left(u_{i_{0}}\right) \neq 0$.

(b) This follows directly from part (a). 
Part (b) of the above implies that $\Theta_{X}\left(C^{\omega}\right)$ is a basis for $\bigotimes_{i \in I}^{\omega} X_{i}$, where $C^{\omega}$ is as defined in the following result.

Corollary 2.7. (a) Let c $: \Omega_{I ; X} \rightarrow \Pi_{i \in I} X_{i}^{\times}$be a cross section. For each $\omega \in \Omega_{I ; X}$ and $i \in I$, we pick a basis $B_{i}^{\omega}$ of $X_{i}$ that contains $c(\omega)_{i}$ and set

$$
C^{\omega}:=\left\{x \in \Pi_{i \in I}^{\omega} X_{i}: x_{i} \in B_{i}^{\omega}, \forall i \in I\right\} .
$$

If $C:=\bigcup_{\omega \in \Omega_{I ; X}} C^{\omega}$, then $\Theta_{X}(C)$ is a basis for $\bigotimes_{i \in I} X_{i}$.

(b) If $\Phi_{i}: X_{i} \rightarrow Y_{i}$ is an injective linear map $(i \in I)$, the induced linear map $\bigotimes_{i \in I} \Phi_{i}: \bigotimes_{i \in I} X_{i} \rightarrow \bigotimes_{i \in I} Y_{i}$ is injective.

Proposition 2.8. The map $\Psi: \bigotimes_{i \in I} L\left(X_{i} ; Y_{i}\right) \rightarrow L\left(\bigotimes_{i \in I} X_{i} ; \bigotimes_{i \in I} Y_{i}\right)$ (given by the universal property) is injective.

Proof. Suppose that $T^{(1)}, \ldots, T^{(n)} \in \Pi_{i \in I} L\left(X_{i} ; Y_{i}\right)^{\times}$are mutually inequivalent elements (under $\sim$ ), $F \in \mathfrak{F}, R^{(1)}, \ldots, R^{(n)} \in \bigotimes_{i \in F} L\left(X_{i} ; Y_{i}\right)$ with $S^{(k)}:=J_{F}^{T^{(k)}}\left(R^{(k)}\right)$ $(k=1, \ldots, n)$ satisfying

$$
\Psi\left(\sum_{k=1}^{n} S^{(k)}\right)=0
$$

Using an induction argument, it suffices to show that $S^{(1)}=0$.

If $n=1$, we take any $x \in \Pi_{i \in I} X_{i}^{\times}$with $T_{i}^{(1)} x_{i} \neq 0(i \in I)$. If $n>1$, we claim that there is $x \in \Pi_{i \in I} X_{i}^{\times}$such that

$$
\left[T_{i}^{(1)} x_{i}\right]_{i \in I} \in \Pi_{i \in I} Y_{i}^{\times} \quad \text { and } \quad\left[T_{i}^{(k)} x_{i}\right]_{i \in I} \nsim\left[T_{i}^{(1)} x_{i}\right]_{i \in I} \quad(k=2, \ldots, n) .
$$

In fact, let $I^{k}:=\left\{i \in I: T_{i}^{(k)} \neq T_{i}^{(1)}\right\}$, which is an infinite set for any $k=2, \ldots, n$. For any $i \in I$, we put $N_{i}:=\left\{k \in\{2, \ldots, n\}: i \in I^{k}\right\}$ and take any $x_{i} \in$ $X_{i} \backslash\left(\cup_{k \in N_{i}} \operatorname{ker}\left(T_{i}^{(k)}-T_{i}^{(1)}\right) \cup \operatorname{ker} T_{i}^{(1)}\right)$ (note that $X_{i}$ cannot be a finite union of proper subspaces). Thus, $T_{i}^{(1)} x_{i} \neq 0$ (for each $i \in I$ ) and $T_{i}^{(k)} x_{i} \neq T_{i}^{(1)} x_{i}$ (for $k \in\{2, \ldots, n\}$ and $\left.i \in I^{k}\right)$.

Now, we have

$$
\Psi\left(S^{(1)}\right)\left(\bigotimes_{i \in I}^{x} X_{i}\right) \cap\left(\sum_{k=2}^{n} \Psi\left(S^{(k)}\right)\left(\bigotimes_{i \in I}^{x} X_{i}\right)\right)=(0)
$$

by Theorem 2.5 and the fact that $\Psi\left(S^{(l)}\right)\left(\bigotimes_{i \in I}^{x} X_{i}\right) \in \bigotimes_{i \in I}^{y^{(l)}} Y_{i}$, where $y_{i}^{(l)}=T_{i}^{(l)} x_{i}$ $(i \in I ; l=1, \ldots, n)$. Consequently, $\left.\Psi\left(S^{(1)}\right)\right|_{\bigotimes_{i \in I}^{x} X_{i}}=0$. As $T_{i}^{(1)} x_{i} \neq 0(i \in I)$, it is easy to see that $R^{(1)}=0$ as required.

Note that $\Psi$ is not surjective even if $X_{i}=Y_{i}=\mathbb{C}(i \in I)$ since in this case, $\Psi$ is a homomorphism and $\bigotimes_{i \in I} \mathbb{C}$ is commutative while $L\left(\bigotimes_{i \in I} \mathbb{C}\right)$ is not.

The following result follows from Propositions 2.3 (c) and 2.8 as well as Corollary 2.7 (b). It says that an infinite tensor product of vector spaces is automatically a faithful module over a big commutative algebra. 
Corollary 2.9. If $X_{i}$ is a faithful $A_{i}$-module $(i \in I)$, then $\bigotimes_{i \in I} X_{i}$ is a faithful $\bigotimes_{i \in I} A_{i}$-module. In particular, $\bigotimes_{i \in I} Y_{i}$ is a faithful unital $\mathbb{C}^{\otimes I}$-module.

Example 2.10. (a) If $\beta \in \Pi_{i \in I} \mathbb{C}^{\times}$, then $\bigotimes_{i \in I}^{\beta} \mathbb{C}=\mathbb{C} \cdot \otimes_{i \in I} \beta_{i}$. In fact, for any $F \in \mathfrak{F}$ and $\mu_{i} \in \mathbb{C}(i \in F)$, we have $J_{F}^{\beta}\left(\otimes_{i \in F} \mu_{i}\right)=\left(\Pi_{i \in F} \mu_{i} / \beta_{i}\right)\left(\otimes_{i \in I} \beta_{i}\right)$.

(b) For $n \in \mathbb{N}$, let $I_{1}, \ldots, I_{n}$ be infinite disjoint subsets of $I$ with $I=\bigcup_{k=1}^{n} I_{k}$ and $\bar{\beta}=\left(\beta_{1}, \ldots, \beta_{n}\right) \in\left(\mathbb{C}^{\times}\right)^{n}$. Define $\widetilde{\beta} \in \Pi_{i \in I} \mathbb{C}^{\times}$by $\widetilde{\beta}_{i}=\beta_{k}$ whenever $i \in I_{k}$. Then $\bar{\beta} \mapsto[\widetilde{\beta}]_{\sim}$ is an injective group homomorphism from $\left(\mathbb{C}^{\times}\right)^{n}$ to $\Omega_{I ; \mathbb{C}}$.

(c) Let $G$ be a subgroup of $\mathbb{T}^{n} \subseteq\left(\mathbb{C}^{\times}\right)^{n}$ (where $\mathbb{T}:=\{t \in \mathbb{C}:|t|=1\}$ ). If $\overline{\beta^{(1)}}, \ldots, \overline{\beta^{(m)}}$ are distinct elements in $G$ and $\widetilde{\beta^{(1)}}, \ldots, \widetilde{\beta^{(m)}} \in \Pi_{i \in I} \mathbb{C}^{\times}$are as in part (b), then $\otimes_{i \in I} \widetilde{\beta_{i}^{(1)}}, \ldots, \otimes_{i \in I} \widetilde{\beta_{i}^{(m)}}$ are linearly independent in $\mathbb{C}^{\otimes I}$. Therefore, the ${ }^{*}$-subalgebra of $\mathbb{C}^{\otimes I}$ generated by $\left\{\otimes_{i \in I} \widetilde{\beta}_{i}: \bar{\beta} \in G\right\}$ is ${ }^{*}$-isomorphic to the group algebra $\mathbb{C}[G]$.

As $\otimes_{i \in I} \alpha_{i}=\left(\Pi_{i \in I} \alpha_{i}\right)\left(\otimes_{i \in I} 1\right)$ if $\alpha_{i}=1$ e.f., one may regard $\otimes_{i \in I} \alpha_{i}$ as a generalization of the product. In this case, one can consider infinite products like $(-1)^{I}$.

\section{Tensor products of unital *-algebras}

Throughout this section, $A_{i}$ is a unital ${ }^{*}$-algebra with identity $e_{i}(i \in I)$, and we set $\Omega_{I ; A}^{\mathrm{ut}}:=\Pi_{i \in I} U_{A_{i}} / \sim$.

Notice that in this case, $\Omega_{I ; A}$ is a ${ }^{*}$-semigroup with identity and $\Omega_{I ; A}^{\text {ut }}$ can be regarded as a subgroup of $\Omega_{I ; A}$ with the inverse being the involution on $\Omega_{I ; A}$. Moreover, $\bigotimes_{i \in I} A_{i}$ is a $\Omega_{I ; A}$-graded *-algebra in the sense that for any $\omega, \omega^{\prime} \in \Omega_{I ; A}$,

$$
\left(\bigotimes_{i \in I}^{\omega} A_{i}\right) \cdot\left(\bigotimes_{i \in I}^{\omega^{\prime}} A_{i}\right) \subseteq \bigotimes_{i \in I}^{\omega \omega^{\prime}} A_{i} \text { and }\left(\bigotimes_{i \in I}^{\omega} A_{i}\right)^{*} \subseteq \bigotimes_{i \in I}^{\omega^{*}} A_{i}
$$

By Proposition 2.6(b), $\bigotimes_{i \in I}^{e} A_{i}$ can be identified with the unital *-algebra inductive limit of finite tensor products of $A_{i}$. We will study the following ${ }^{*}$-subalgebra that contains $\bigotimes_{i \in I}^{e} A_{i}$ :

$$
\bigotimes_{i \in I}^{\mathrm{ut}} A_{i}:=\bigoplus_{\omega \in \Omega_{I ; A}^{\mathrm{ut}}} \bigotimes_{i \in I}^{\omega} A_{i}
$$

The motivation for considering this subalgebra is partially Example 3.1 (a) below, and partially because it has good representations (see the discussion after Proposition 5.1 below). Moreover, if all the $A_{i}$ are linear spans of $U_{A_{i}}$ (in particular, if they are $C^{*}$-algebras or group algebras), then $\bigotimes_{i \in I}^{\text {ut }} A_{i}$ is the linear span of $\Theta_{A}\left(\Pi_{i \in I} U_{A_{i}}\right)$. If $A_{i}=A$ for all $i \in I$, we write $A_{\mathrm{ut}}^{\otimes I}:=\bigotimes_{i \in I}^{\mathrm{ut}} A_{i}$. 
Example 3.1. (a) Let $G_{i}$ be a group and $\mathbb{C}\left[G_{i}\right]$ be its group algebra $(i \in I)$. If $\Lambda: \Pi_{i \in I} G_{i} \rightarrow \Pi_{i \in I} U_{\mathbb{C}\left[G_{i}\right]}$ is the canonical map, then $\lambda:=\Theta_{\mathbb{C}[G]} \circ \Lambda$ gives a *-isomorphism from $\mathbb{C}\left[\Pi_{i \in I} G_{i}\right]$ to the ${ }^{*}$-subalgebra

$$
\bigotimes_{i \in I}^{\Lambda\left(\Pi_{i} \in I G_{i}\right)} \mathbb{C}\left[G_{i}\right]:=\sum_{t \in \Pi_{i \in I} G_{i}} \bigotimes_{i \in I}^{\Lambda(t)} \mathbb{C}\left[G_{i}\right] \subseteq \bigotimes_{i \in I}^{\mathrm{ut}} \mathbb{C}\left[G_{i}\right]
$$

In fact, $\lambda$ induces a ${ }^{*}$-homomorphism from $\mathbb{C}\left[\Pi_{i \in I} G_{i}\right]$ to $\bigotimes_{i \in I}^{\text {ut }} \mathbb{C}\left[G_{i}\right]$. Let $q$ : $\Pi_{i \in I} G_{i} \rightarrow \prod_{i \in I} G_{i} / \oplus_{i \in I} G_{i}$ be the quotient map. For a fixed $s \in \prod_{i \in I} G_{i}$, if we set

$$
\bigoplus_{i \in I}^{s} G_{i}:=\left\{t \in \Pi_{i \in I} G_{i}: q(t)=q(s)\right\},
$$

then $s^{-1}\left(\bigoplus_{i \in I}^{s} G_{i}\right)=\bigoplus_{i \in I} G_{i}$. Thus, $\left\{\lambda(t): t \in \bigoplus_{i \in I}^{s} G_{i}\right\}$ is a set of linearly independent elements in $\bigotimes_{i \in I} \mathbb{C}\left[G_{i}\right]\left(\right.$ as $\left.\lambda\right|_{\mathbb{C}\left[\oplus_{i \in I} G_{i}\right]}$ is a bijection onto $\left.\bigotimes_{i \in I}^{e} \mathbb{C}\left[G_{i}\right]\right)$. On the other hand, if $s^{(1)}, \ldots, s^{(n)} \in \Pi_{i \in I} G_{i}$ are such that $q\left(s^{(k)}\right) \neq q\left(s^{(l)}\right)$ whenever $k \neq l$, then $\lambda\left(s^{(1)}\right), \ldots, \lambda\left(s^{(n)}\right)$ are linearly independent in $\bigotimes_{i \in I} \mathbb{C}\left[G_{i}\right]$ (see Theorem 2.5). Consequently, $\left\{\lambda(t): t \in \Pi_{i \in I} G_{i}\right\}$ form a basis for $\bigotimes_{i \in I}^{\Lambda\left(\Pi_{i \in I} G_{i}\right)} \mathbb{C}\left[G_{i}\right]$.

(b) It is well known that there is a twisted action $(\alpha, u)$, in the sense of Busby and Smith, of $\Omega_{I ; G}:=\Pi_{i \in I} G_{i} / \oplus_{i \in I} G_{i}$ on $\mathbb{C}\left[\bigoplus_{i \in I} G_{i}\right] \cong \bigotimes_{i \in I}^{e} \mathbb{C}\left[G_{i}\right]$ (see 2.1 in [5]) such that $\mathbb{C}\left[\Pi_{i \in I} G_{i}\right]$ is *-isomorphic to the algebraic crossed product $\bigotimes_{i \in I}^{e} \mathbb{C}\left[G_{i}\right] \rtimes_{\alpha, u} \Omega_{I ; G}$.

There exists a canonical action $\Xi$ of $\prod_{i \in I} U_{A_{i}}$ on $\bigotimes_{i \in I}^{\text {ut }} A_{i}$ given by inner automorphisms, i.e.

$$
\Xi_{u}(a):=\left(\otimes_{i \in I} u_{i}\right) \cdot a \cdot\left(\otimes_{i \in I} u_{i}^{*}\right) \quad\left(u \in \Pi_{i \in I} U_{A_{i}} ; a \in \bigotimes_{i \in I}^{\mathrm{ut}} A_{i}\right) .
$$

This induces an action $\Xi^{e}$ of $\prod_{i \in I} U_{A_{i}}$ on the subalgebra $\bigotimes_{i \in I}^{e} A_{i}$. The following result gives an identification of $\bigotimes_{i \in I}^{\mathrm{ut}} A_{i}$ as the algebraic crossed product (see, e.g., page 166 of [16]) of a cocycle twisted action (i.e., a twisted action in the sense of Busby and Smith) of $\Omega_{I ; A}^{\mathrm{ut}}$ on $\bigotimes_{i \in I}^{e} A_{i}$ induced by $\Xi^{e}$.

Before we give this result, let us recall that an abelian group $G$ is divisible if for any $g \in G$ and $n \in \mathbb{N}$, there is $h \in G$ with $g=h^{n}$.

Theorem 3.2. (a) There is a cocycle twisted action $(\check{\Xi}, m)$ of $\Omega_{I ; A}^{\mathrm{ut}}$ on $\bigotimes_{i \in I}^{e} A_{i}$ such that $\bigotimes_{i \in I}^{\mathrm{ut}} A_{i}$ is $\Omega_{I ; A}^{\mathrm{ut}}$-graded ${ }^{*}$-isomorphic to $\left(\bigotimes_{i \in I}^{e} A_{i}\right) \rtimes_{\Xi, m} \Omega_{I ; A}^{\mathrm{ut}}$.

(b) Suppose that all the $A_{i}$ are commutative. If $\bigotimes_{i \in I}^{e} A_{i}$ is a unital ${ }^{*}$-subalgebra of a commutative ${ }^{*}$-algebra $B$ with $U_{B}$ being divisible, $\bigotimes_{i \in I}^{\mathrm{ut}} A_{i}$ is $\Omega_{I ; A}^{\mathrm{ut}}$-graded ${ }^{*}$-isomorphic to a unital ${ }^{*}$-subalgebra of $B \otimes \mathbb{C}\left[\Omega_{I ; A}^{\mathrm{ut}}\right]$. If $U_{\bigotimes_{i \in I}^{e} A_{i}}$ is itself divisible, $\bigotimes_{i \in I}^{\mathrm{ut}} A_{i} \cong\left(\bigotimes_{i \in I}^{e} A_{i}\right) \otimes \mathbb{C}\left[\Omega_{I ; A}^{\mathrm{ut}}\right]$ as $\Omega_{I ; A}^{\mathrm{ut}}$-graded ${ }^{*}$-algebras.

Proof. Let $c: \Omega_{I ; A}^{\mathrm{ut}} \rightarrow \Pi_{i \in I} U_{A_{i}}$ be a cross section with $c\left([e]_{\sim}\right)=e$.

(a) For any $\mu, \nu \in \Omega_{I ; A}^{\text {ut }}$, we set

$$
\check{\Xi}_{\mu}:=\Xi_{c(\mu)}^{e} \text { and } m(\mu, \nu):=\otimes_{i \in I} c(\mu)_{i} c(\nu)_{i} c(\mu \nu)_{i}^{-1} \text {. }
$$


As $c(\mu) c(\nu) \sim c(\mu \nu)$, we have $m(\mu, \nu) \in \bigotimes_{i \in I}^{e} A_{i}$. It is easy to check that $(\check{\Xi}, m)$ is a twisted action in the sense of Busby and Smith. Furthermore, we define $\Psi:\left(\bigotimes_{i \in I}^{e} A_{i}\right) \rtimes_{\check{\Xi}, m} \Omega_{I ; A}^{\mathrm{ut}} \rightarrow \bigotimes_{i \in I}^{\mathrm{ut}} A_{i}$ by

$$
\Psi(f):=\sum_{\omega \in \Omega_{I ; A}^{\mathrm{ut}}} f(\omega)\left(\otimes_{i \in I} c(\omega)_{i}\right) \quad\left(f \in\left(\bigotimes_{i \in I}^{e} A_{i}\right) \rtimes_{\Xi, m} \Omega_{I ; A}^{\mathrm{ut}}\right) .
$$

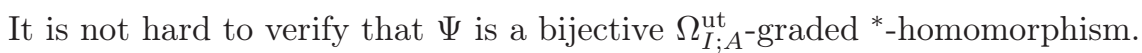

(b) Let $\Pi_{i \in I}^{e} U_{A_{i}}:=\Pi_{i \in I}^{e} A_{i} \cap \Pi_{i \in I} U_{A_{i}}$. By Baer's theorem, $\left.\Theta_{A}\right|_{\Pi_{i \in I}^{e} U_{A_{i}}}$ can be extended to a group homomorphism $\varphi: \Pi_{i \in I} U_{A_{i}} \rightarrow U_{B}$. Since

$$
\varphi(c(\mu)) \varphi(c(\nu)) \varphi(c(\mu \nu))^{-1}=\otimes_{i \in I} c(\mu)_{i} c(\nu)_{i} c(\mu \nu)_{i}^{-1} \quad\left(\mu, \nu \in \Omega_{I ; A}^{\mathrm{ut}}\right),
$$

the map $\Phi: \bigotimes_{i \in I}^{\mathrm{ut}} A_{i} \rightarrow B \otimes \mathbb{C}\left[\Omega_{I ; A}^{\mathrm{ut}}\right]$ given by

$$
\Phi(a):=\left(a \cdot \otimes_{i \in I} c(\omega)_{i}^{-1}\right) \varphi(c(\omega)) \otimes \lambda(\omega) \quad\left(a \in \bigotimes_{i \in I}^{\omega} A_{i} ; \omega \in \Omega_{I ; A}^{\mathrm{ut}}\right)
$$

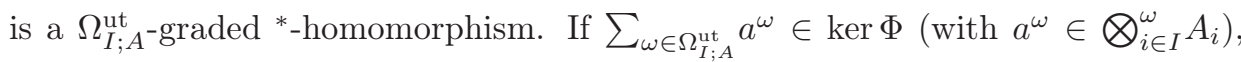
then for every $\omega \in \Omega_{I ; A}^{\text {ut }}$, one has $\left(a^{\omega} \cdot \otimes_{i \in I} c(\omega)_{i}^{-1}\right) \varphi(c(\omega))=0$, which implies $a^{\omega}=0$, and hence $\Phi$ is injective. The image of $\Phi$ is the linear span of

$$
\left\{b \varphi(c(\omega)) \otimes \lambda(\omega): b \in \bigotimes_{i \in I}^{e} A_{i} ; \omega \in \Omega_{I ; A}^{\mathrm{ut}}\right\},
$$

and it is clear that $\Phi$ is surjective if $B=\bigotimes_{i \in I}^{e} A_{i}$.

Remark 3.3. (a) The cocycle twisted action $(\check{\Xi}, m)$ depends on the choice of a cross section, and different cross sections may give different twisted actions (although their crossed products are all isomorphic). On the other hand, the map $\Phi$ in part (b) also depends on the choice of a cross section as well as the choice of an extension of $\left.\Theta_{A}\right|_{\Pi_{i \in I}^{e}} U_{A_{i}}$.

(b) If $S_{i}$ is a set and $A_{i}$ is a ${ }^{*}$-subalgebra of $\ell^{\infty}\left(S_{i}\right)(i \in I)$, then by Theorem $3.2(\mathrm{~b}), \bigotimes_{i \in I}^{\mathrm{ut}} A_{i}$ is a ${ }^{*}$-subalgebra of $\ell^{\infty}\left(\Pi_{i \in I} S_{i}\right) \otimes \mathbb{C}\left[\Omega_{I ; A}^{\text {ut }}\right]$. Our first proof for this fact use 18.4 in [6] and 7.1 in [7].

(c) If all the $A_{i}$ are commutative, then $\bigotimes_{i \in I}^{\mathrm{ut}} A_{i} \cong\left(\otimes_{i \in I^{e}}^{e} A_{i}\right) \otimes \mathbb{C}\left[\Omega_{I ; A}^{\mathrm{ut}}\right]$ as $\Omega_{I ; A^{-}}^{\mathrm{ut}}$ graded *-algebras if and only if there is a group homomorphism $\pi: \Omega_{I ; A}^{\text {ut }} \rightarrow U_{\bigotimes_{i \in I}^{\text {ut }} A_{i}}$ such that $\pi(\omega) \in \bigotimes_{i \in I}^{\omega} A_{i}\left(\omega \in \Omega_{I ; A}^{u t}\right)$. In fact, if such a $\pi$ exists, one may replace $\left(a \cdot \otimes_{i \in I} c(\omega)_{i}^{-1}\right) \varphi(c(\omega))$ in $(3.2)$ with $a \pi\left(\omega^{-1}\right)$ and show that the corresponding $\Phi$ is a *-isomorphism.

Clearly, the second statement of Theorem $3.2(\mathrm{~b})$ applies to the case when $A_{i}=\mathbb{C}^{n_{i}}$ for some $n_{i} \in \mathbb{N}(i \in I)$. In particular, Theorem $3.2(\mathrm{~b})$ and its argument give the following corollary.

Corollary 3.4. If $\varphi_{1}$ is as in Example 2.2 (a) and $\varphi: \Pi_{i \in I} \mathbb{T} \rightarrow \mathbb{T}$ is a group homomorphism that extends $\left.\varphi_{1}\right|_{\Pi_{i \in I}^{1} \mathbb{T}}$ (its existence is guaranteed by Baer's theorem), then $\Phi\left(\otimes_{i \in I} \alpha_{i}\right):=\varphi(\alpha) \lambda\left([\alpha]_{\sim}\right)\left(\alpha \in \Pi_{i \in I} \mathbb{T}\right)$ is a well-defined ${ }^{*}$-isomorphism from $\mathbb{C}_{\mathrm{ut}}^{\otimes I}$ onto $\mathbb{C}\left[\Omega_{I ; \mathbb{C}}^{\mathrm{ut}}\right]$. 
Conversely, it is clear that if $\varphi: \Pi_{i \in I} \mathbb{T} \rightarrow \mathbb{T}$ is any map such that $\Phi$ as defined in the above is a well-defined ${ }^{*}$-isomorphism, then $\varphi$ is a group homomorphism extending $\left.\varphi_{1}\right|_{\Pi_{i \in I}^{1} \mathbb{T}}$. On the other hand, there is a simpler proof for Corollary 3.4. In fact, for $\alpha, \beta \in \Pi_{i \in I} \mathbb{T}$ with $\alpha \sim \beta$, one has $\varphi(\alpha)^{-1} \cdot \otimes_{i \in I} \alpha_{i}=\varphi(\beta)^{-1} \cdot \otimes_{i \in I} \beta_{i}$. Thus, $[\alpha]_{\sim} \mapsto \varphi(\alpha)^{-1} \cdot \otimes_{i \in I} \alpha_{i}$ is a well-defined group homomorphism from $\Omega_{I ; \mathbb{C}}^{\text {ut }}$ to $U_{\mathbb{C}_{\mathrm{ut}}^{\otimes I}}$ such that $\left\{\varphi(\alpha)^{-1} \cdot \otimes_{i \in I} \alpha_{i}:[\alpha]_{\sim} \in \Omega_{I ; \mathbb{C}}^{\mathrm{ut}}\right\}$ is a basis for $\mathbb{C}_{\mathrm{ut}}^{\otimes I}$.

Example 3.5. For any subgroup $G \subseteq \mathbb{T}^{n}$, the algebra defined as in Example $2.10(\mathrm{c})$ is a ${ }^{*}$-subalgebras of $\mathbb{C}_{\mathrm{ut}}^{\otimes I}$.

In the remainder of this section, we will show that the center of $\bigotimes_{i \in I}^{\text {ut }} A_{i}$ is the tensor product of centers of the $A_{i}$ when $A_{i}=\operatorname{span} U_{A_{i}}$ for all $i \in I$.

If $A$ is an algebra and $G$ is a group, we denote by $Z(A)$ and $Z(G)$ the center of $A$ and the center of $G$ respectively. Clearly, the inclusion $\Pi_{i \in I} U_{Z\left(A_{i}\right)} \subseteq \Pi_{i \in I} U_{A_{i}}$ induces an injective group homomorphism from $\Omega_{I ; Z(A)}^{\mathrm{ut}}$ to $\Omega_{I ; A}^{\mathrm{ut}}$ and we regard the former as a subgroup of the latter.

Theorem 3.6. Suppose that there is $F_{0} \in \mathfrak{F}$ with $A_{i}=\operatorname{span} U_{A_{i}}$ for all $i \in I_{0}:=$ $I \backslash F_{0}$.

(a) $Z\left(\Omega_{I ; A}^{\mathrm{ut}}\right)=\Omega_{I ; Z(A)}^{\mathrm{ut}}$. Moreover, $Z\left(\Omega_{I ; A}^{\mathrm{ut}}\right)=\Omega_{I ; A}^{\mathrm{ut}}$ if and only if all but a finite number of the $A_{i}$ are commutative.

(b) Every element in $\Omega_{I ; A}^{\mathrm{ut}} \backslash Z\left(\Omega_{I ; A}^{\mathrm{ut}}\right)$ has an infinite conjugacy class.

(c) $Z\left(\bigotimes_{i \in I}^{\mathrm{ut}} A_{i}\right)=\bigotimes_{i \in I}^{\mathrm{ut}} Z\left(A_{i}\right)$.

Proof. (a) It is obvious that $\Omega_{I ; Z(A)}^{\mathrm{ut}} \subseteq Z\left(\Omega_{I ; A}^{\mathrm{ut}}\right)$. Suppose $u \in \Pi_{i \in I} U_{A_{i}}$ with $[u]_{\sim} \notin \Omega_{I ; Z(A)}^{\mathrm{ut}}$. There is an infinite subset $J \subseteq I_{0}$ such that $u_{i} \notin Z\left(A_{i}\right)(i \in J)$. For each $i \in J$, one can find $v_{i} \in U_{A_{i}}$ such that $u_{i} v_{i} \neq v_{i} u_{i}$. For any $i \in I \backslash J$, we put $v_{i}=e_{i}$. Then $[v]_{\sim} \in \Omega_{I ; A}^{\mathrm{ut}}$ and $[u]_{\sim}[v]_{\sim} \neq[v]_{\sim}[u]_{\sim}$. Consequently, $[u]_{\sim} \notin Z\left(\Omega_{I ; A}^{\mathrm{ut}}\right)$. This argument also shows that if the set $\left\{i \in I: Z\left(A_{i}\right) \neq A_{i}\right\}$ is infinite, then $Z\left(\Omega_{I ; A}^{\mathrm{ut}}\right) \neq \Omega_{I ; A}^{\mathrm{ut}}$. Conversely, it is clear that $\Omega_{I ; Z(A)}^{\mathrm{ut}}=\Omega_{I ; A}^{\mathrm{ut}}$ if all but a finite numbers of the $A_{i}$ are commutative.

(b) Suppose that $[u]_{\sim} \in \Omega_{I ; A}^{\mathrm{ut}} \backslash Z\left(\Omega_{I ; A}^{\mathrm{ut}}\right)$ and $\left\{i_{n}\right\}_{n \in \mathbb{N}}$ is a sequence of distinct elements in $I_{0}$ such that $u_{i_{n}} \notin Z\left(A_{i_{n}}\right)(n \in \mathbb{N})$. For each $n \in \mathbb{N}$, choose $v_{i_{n}} \in U_{A_{i_{n}}}$ with $v_{i_{n}} u_{i_{n}} v_{i_{n}}^{*} \neq u_{i_{n}}$. For any prime number $p$, we set $w_{i_{n}}^{(p)}:=v_{i_{n}}(n \in \mathbb{N} p)$, and $w_{i}^{(p)}:=e_{i}$ if $i \in I \backslash\left\{i_{n}: n \in \mathbb{N} p\right\}$. If $p$ and $q$ are distinct prime numbers, then

$$
w_{i_{n}}^{(q)} u_{i_{n}}\left(w_{i_{n}}^{(q)}\right)^{*}=u_{i_{n}} \neq w_{i_{n}}^{(p)} u_{i_{n}}\left(w_{i_{n}}^{(p)}\right)^{*} \quad(n \in \mathbb{N} p \backslash \mathbb{N} q) .
$$

Consequently, $w^{(q)} u\left(w^{(q)}\right)^{*} \nsim w^{(p)} u\left(w^{(p)}\right)^{*}$, and the conjugacy class of $[u]_{\sim}$ is infinite.

(c) Since $Z\left(\bigotimes_{i \in I}^{\mathrm{ut}} A_{i}\right)=\bigotimes_{i \in F_{0}} Z\left(A_{i}\right) \otimes Z\left(\bigotimes_{i \in I_{0}}^{\mathrm{ut}} A_{i}\right)$, we may assume that $A_{i}=$ $\operatorname{span} U_{A_{i}}$ for all $i \in I$. In this case, $Z\left(\otimes_{i \in I}^{\mathrm{ut}} A_{i}\right)=\left(\bigotimes_{i \in I}^{\mathrm{ut}} A_{i}\right)^{\Xi}$, where $\left(\bigotimes_{i \in I}^{\mathrm{ut}} A_{i}\right)^{\Xi}$ is the fixed point algebra of the action $\Xi$ as defined above. Moreover, one has $\bigotimes_{i \in I}^{\text {ut }} Z\left(A_{i}\right) \subseteq Z\left(\bigotimes_{i \in I}^{\text {ut }} A_{i}\right)$ and it remains to show that $\left(\bigotimes_{i \in I}^{\text {ut }} A_{i}\right)^{\Xi} \subseteq \bigotimes_{i \in I}^{\text {ut }} Z\left(A_{i}\right)$. 
Let $v^{(1)}, \ldots, v^{(n)} \in \Pi_{i \in I} U_{A_{i}}$ be mutually inequivalent elements, let $F \in \mathfrak{F}$, and let $b_{1}, \ldots, b_{n} \in \bigotimes_{i \in F} A_{i} \backslash\{0\}$ be such that $a:=\sum_{k=1}^{n} J_{F}^{v^{(k)}}\left(b_{k}\right) \in\left(\bigotimes_{i \in I}^{\text {ut }} A_{i}\right)^{\Xi}$. We first claim that $\left[v^{(k)}\right]_{\sim} \in \Omega_{I ; Z(A)}^{\text {ut }}(k=1, \ldots, n)$. Suppose, to the contrary, that $\left[v^{(1)}\right]_{\sim} \notin \Omega_{I ; Z(A)}^{\mathrm{ut}}=Z\left(\Omega_{I ; A}^{\mathrm{ut}}\right)$. For every $u \in \Pi_{i \in I} U_{A_{i}}$, one has

$$
\Xi_{u}\left(J_{F}^{v^{(1)}}\left(b_{k}\right)\right) \in\left(\bigotimes_{i \in I}^{\left[u v^{(1)} u^{*}\right] \sim} A_{i}\right) \backslash\{0\} .
$$

As $\Xi_{u}(a)=a$, we see that $\left[u v^{(1)} u^{*}\right]_{\sim} \in\left\{\left[v^{(1)}\right]_{\sim}, \ldots,\left[v^{(n)}\right]_{\sim}\right\}$, which contradicts the fact that $\left\{\left[u v^{(1)} u^{*}\right]_{\sim}:[u]_{\sim} \in \Omega_{I ; A}^{\mathrm{ut}}\right\}$ is an infinite set (by part (b)).

By enlarging $F$, we may assume that $v^{(k)} \in \Pi_{i \in I} U_{Z\left(A_{i}\right)}(k=1, \ldots, n)$. For each $u \in \Pi_{i \in I} U_{A_{i}}$ and $k \in\{1, \ldots, n\}$, one has $\Xi_{u}\left(J_{F}^{v^{(k)}}\left(b_{k}\right)\right)=J_{F}^{v^{(k)}}\left(b_{k}\right)$ and so, $b_{k} \in Z\left(\bigotimes_{i \in F} A_{i}\right)$. Therefore, $a \in \bigotimes_{i \in I}^{\mathrm{ut}} Z\left(A_{i}\right)$, as expected.

The reader should notice that $\bigotimes_{i \in I}^{\mathrm{ut}} Z\left(A_{i}\right)$ equals $\bigoplus_{\omega \in Z\left(\Omega_{I: \mathrm{A}}^{\mathrm{ut}}\right)} \bigotimes_{i \in I}^{\omega} Z\left(A_{i}\right)$ instead of $\bigoplus_{\omega \in \Omega_{I: A}^{\mathrm{ut}}} \bigotimes_{i \in I}^{\omega} Z\left(A_{i}\right)$ (strictly speaking, the latter object does not make sense).

Example 3.7. (a) If $n_{i} \in \mathbb{N}(i \in I)$, then $Z\left(\bigotimes_{i \in I}^{\mathrm{ut}} M_{n_{i}}(\mathbb{C})\right) \cong \mathbb{C}_{\mathrm{ut}}^{\otimes I}$.

(b) If $G_{i}$ are icc groups, then $Z\left(\bigotimes_{i \in I}^{\mathrm{ut}} \mathbb{C}\left[G_{i}\right]\right) \cong \mathbb{C}_{\mathrm{ut}}^{\otimes I}$ canonically.

We end this section with the following brief discussion on the non-unital case. Suppose that $\left\{A_{i}\right\}_{i \in I}$ is a family of *-algebras, not necessarily unital. If $M\left(A_{i}\right)$ is the double centraliser algebra of $A_{i}(i \in I)$, we define an ideal, $\bigotimes_{i \in I}^{\text {ut }} A_{i}$, of $\bigotimes_{i \in I}^{\mathrm{ut}} M\left(A_{i}\right)$ as follows:

$$
\bigotimes_{i \in I}^{\mathrm{ut}} A_{i}:=\operatorname{span}\left\{J_{F}^{u}(a): F \in \mathfrak{F} ; a \in \bigotimes_{i \in F} A_{i} ; u \in \Pi_{i \in I} U_{M\left(A_{i}\right)}\right\} .
$$

In general, $\bigotimes_{i \in I}^{\mathrm{ut}} A_{i}$ is not a subset of $\bigotimes_{i \in I} A_{i}$. In a similar fashion, we define

$$
\bigotimes_{i \in I}^{e} A_{i}:=\operatorname{span}\left\{J_{F}^{u}(a): F \in \mathfrak{F} ; a \in \bigotimes_{i \in F} A_{i} ; u \in \Pi_{i \in I} U_{M\left(A_{i}\right)} ; u \sim e\right\},
$$

which is an ideal of $\bigotimes_{i \in I}^{e} M\left(A_{i}\right)$. By the proof of Theorem 3.2 (a), one may identify $\bigotimes_{i \in I}^{\mathrm{ut}} A_{i}$ as the ideal of $\left(\bigotimes_{i \in I}^{e} M\left(A_{i}\right)\right) \rtimes_{\Xi, m} \Omega_{I ; M(A)}^{\mathrm{ut}}$ consisting of functions from $\Omega_{I ; M(A)}^{\mathrm{ut}}$ to $\bigotimes_{i \in I}^{e} A_{i}$ having finite supports.

\section{Tensor products of inner product spaces}

Throughout this section, $\left(H_{i},\langle\cdot, \cdot\rangle\right)$ is a non-zero inner product space $(i \in I)$. Moreover, we denote $\Omega_{I ; H}^{\text {unit }}:=\Pi_{i \in I} \mathfrak{S}_{1}\left(H_{i}\right) / \sim$.

If $B$ is a unital *-algebra and $X$ is a unital left $B$-module, a map $\langle\cdot, \cdot\rangle_{B}: X \times$ $X \rightarrow B$ is called a (left) Hermitian $B$-form on $X$ if $\langle a x+y, z\rangle_{B}=a\langle x, z\rangle_{B}+\langle y, z\rangle_{B}$ and $\langle x, y\rangle_{B}^{*}=\langle y, x\rangle_{B}(x, y, z \in X ; a \in B)$. It is easy to see that a Hermitian $B$-form on $X$ can be regarded as a $B$-bimodule map $\theta: X \otimes \tilde{X} \rightarrow B$ satisfying $\theta(x \otimes \tilde{y})^{*}=\theta(y \otimes \tilde{x})$ (where $\tilde{X}$ is the conjugate vector space of $X$ regarded as a 
unital right $B$-module in the canonical way). Consequently, part (a) of the following result follows readily from the universal property of tensor products, while part (b) is easily verified.

Proposition 4.1. (a) There is a Hermitian $\mathbb{C}^{\otimes I}$-form on $\bigotimes_{i \in I} H_{i}$ such that $\left\langle\otimes_{i \in I} x_{i}, \otimes_{i \in I} y_{i}\right\rangle_{\mathbb{C} \otimes I}:=\otimes_{i \in I}\left\langle x_{i}, y_{i}\right\rangle\left(x, y \in \Pi_{i \in I} H_{i}\right)$.

(b) For a fixed $\mu \in \Omega_{I ; H}^{\text {unit }}$, one has $\left\langle\Theta_{H}(x), \Theta_{H}(y)\right\rangle_{\mathbb{C} \otimes I}=\Pi_{i \in I}\left\langle x_{i}, y_{i}\right\rangle\left(\otimes_{i \in I} 1\right)$ $\left(x, y \in \Pi_{i \in I}^{\mu} H_{i}\right)$. This induces an inner product on $\bigotimes_{i \in I}^{\mu} H_{i}$ that coincides with the one given by the inductive limit of $\left(\bigotimes_{i \in F} H_{i}, J_{G ; F}^{\mu}\right)_{F \subseteq G \in \mathfrak{F}}$, in the category of inner product spaces with isometries as morphisms.

We want to construct a nice inner product space from the above Hermitian $\mathbb{C}^{\otimes I}$-form. A naive idea is to appeal to a construction for Hilbert $C^{*}$-modules that produces a Hilbert space from a positive linear functional on $\mathbb{C}^{\otimes I}$. However, the difficulty is that there is no canonical order structure on $\mathbb{C}^{\otimes I}$. Nevertheless, we will make a similar construction using the functional $\phi_{1}$ in Example 2.2 (a). In this case, one can only consider a subspace of $\bigotimes_{i \in I} H_{i}$ (see Example 4.3 below).

Lemma 4.2. Define $\langle\xi, \eta\rangle_{\phi_{1}}:=\phi_{1}\left(\langle\xi, \eta\rangle_{\mathbb{C} \otimes I}\right)\left(\xi, \eta \in \bigotimes_{i \in I} H_{i}\right)$ and set

$$
\bigotimes_{i \in I}^{\text {ct }} H_{i}:=\operatorname{span} \Theta_{H}\left(\Pi_{i \in I} B_{1}\left(H_{i}\right)\right)
$$

as well as $\bigotimes_{i \in I}^{\text {unit }} H_{i}:=\operatorname{span} \Theta_{H}\left(\prod_{i \in I} \mathfrak{S}_{1}\left(H_{i}\right)\right)$.

(a) For any $\mu \in \Omega_{I ; H}^{\text {unit }}$, the restriction of $\langle\cdot, \cdot\rangle_{\phi_{1}}$ to $\bigotimes_{i \in I}^{\mu} H_{i} \times \bigotimes_{i \in I}^{\mu} H_{i}$ coincides with the inner product in Proposition 4.1 (b).

(b) $\langle\cdot, \cdot\rangle_{\phi_{1}}$ is a positive sesquilinear form on $\bigotimes_{i \in I}^{\mathrm{ct}} H_{i}$ and is an inner product on $\bigotimes_{i \in I}^{\text {unit }} H_{i}$. Moreover, if

$$
K:=\left\{y \in \bigotimes_{i \in I}^{\mathrm{ct}} H_{i}:\langle x, y\rangle_{\phi_{1}}=0, \forall x \in \bigotimes_{i \in I}^{\mathrm{ct}} H_{i}\right\}
$$

then $\bigotimes_{i \in I}^{\mathrm{ct}} H_{i}=K \oplus \bigotimes_{i \in I}^{\mathrm{unit}} H_{i}$ (as vector spaces).

(c) If $I=I_{1} \cup I_{2}$ and $I_{1} \cap I_{2}=\emptyset$, then $\bigotimes_{i \in I}^{\text {unit }} H_{i}=\left(\bigotimes_{i \in I_{1}}^{\text {unit }} H_{i}\right) \otimes\left(\bigotimes_{j \in I_{2}}^{\text {unit }} H_{j}\right)$ as inner product spaces.

Proof. (a) This part is clear.

(b) It is obvious that $\langle\cdot, \cdot\rangle_{\phi_{1}}$ is a sesquilinear form on $\bigotimes_{i \in I}^{\mathrm{ct}} H_{i}$. Let

$$
E:=\left\{x \in \prod_{i \in I} B_{1}\left(H_{i}\right):\left\|x_{i}\right\|<1 \text { for an infinite number of } i \in I\right\}
$$

and $\tilde{K}:=\operatorname{span} \Theta_{H}(E)$. Clearly, $\bigotimes_{i \in I}^{\text {ct }} H_{i}=\tilde{K} \oplus \bigotimes_{i \in I}^{\text {unit }} H_{i}$. Moreover, if $u \in$ $\Pi_{i \in I} B_{1}\left(H_{i}\right)$ and $v \in E$, then $\left\langle u_{i}, v_{i}\right\rangle \neq 1$ for an infinite number of $i \in I$, which implies that $\left\langle\otimes_{i \in I} u_{i}, \otimes_{i \in I} v_{i}\right\rangle_{\phi_{1}}=0$. Consequently, $\tilde{K} \subseteq K$. 
We claim that $\langle\xi, \xi\rangle_{\phi_{1}} \geq 0\left(\xi \in \bigotimes_{i \in I}^{\mathrm{ct}} H_{i}\right)$. Suppose that $\xi=\sum_{k=1}^{n} \lambda_{k} \otimes_{i \in I} u_{i}^{(k)}$ with $\lambda_{1}, \ldots, \lambda_{n} \in \mathbb{C}$ and $u^{(1)}, \ldots, u^{(n)} \in \Pi_{i \in I} B_{1}\left(H_{i}\right)$. Then

$$
\langle\xi, \xi\rangle_{\phi_{1}}=\sum_{k, l=1}^{n} \lambda_{k} \bar{\lambda}_{l} \phi_{1}\left(\otimes_{i \in I}\left\langle u_{i}^{(k)}, u_{i}^{(l)}\right\rangle\right) .
$$

As in the above, $\phi_{1}\left(\otimes_{i \in I}\left\langle u_{i}^{(k)}, u_{i}^{(l)}\right\rangle\right)=0$ if either $u^{(k)}$ or $u^{(l)}$ is in $E$. Thus, by rescaling, we may assume that

$$
u^{(1)}, \ldots, u^{(n)} \in \Pi_{i \in I} \mathfrak{S}_{1}\left(H_{i}\right) .
$$

Furthermore, we assume that there exist $0=n_{0}<\cdots<n_{m}=n$ such that $u^{\left(n_{p}+1\right)} \sim \cdots \sim u^{\left(n_{p+1}\right)}$ for all $p \in\{0, \ldots, m-1\}$, but $u^{\left(n_{p}\right)} \nsim u^{\left(n_{q}\right)}$ whenever $1 \leq$ $p \neq q \leq m$. It is not hard to check that $u^{(k)} \sim u^{(l)}$ if and only if $\left\langle u_{i}^{(k)}, u_{i}^{(l)}\right\rangle=1$ e.f. (as $\left\|u_{i}^{(k)}\right\|,\left\|u_{i}^{(l)}\right\| \leq 1$ ). Consequently, if $1 \leq p \neq q \leq m$,

$$
\phi_{1}\left(\otimes_{i \in I}\left\langle u_{i}^{(k)}, u_{i}^{(l)}\right\rangle\right)=0 \quad \text { when } n_{p}<k \leq n_{p+1} \text { and } n_{q}<l \leq n_{q+1} .
$$

Therefore, in order to show $\langle\xi, \xi\rangle_{\phi_{1}} \geq 0$, it suffices to consider the case when $u^{(k)} \sim u^{(l)}$ for all $k, l \in\{1, \ldots, n\}$, which is the same as $\xi \in \bigotimes_{i \in I}^{u^{(1)}} H_{i}$. Thus, $\langle\xi, \xi\rangle_{\phi_{1}} \geq 0$ by part (a).

Next, we show that $\langle\cdot, \cdot\rangle_{\phi_{1}}$ is an inner product on $\bigotimes_{i \in I}^{\text {unit }} H_{i}$. Suppose that $\xi=\sum_{k=1}^{n} \lambda_{k} \otimes_{i \in I} u_{i}^{(k)}$ with $\lambda_{1}, \ldots, \lambda_{n} \in \mathbb{C}$ and $u^{(1)}, \ldots, u^{(n)} \in \Pi_{i \in I} \mathfrak{S}_{1}\left(H_{i}\right)$ such that $\langle\xi, \xi\rangle_{\phi_{1}}=0$. If $n_{0}, \ldots, n_{m}$ are as above, then

$$
\phi_{1}\left(\left\langle\sum_{k=n_{p}+1}^{n_{p+1}} \lambda_{k} \otimes_{i \in I} u_{i}^{(k)}, \sum_{l=n_{q}+1}^{n_{q+1}} \lambda_{l} \otimes_{i \in I} u_{i}^{(l)}\right\rangle_{\mathbb{C} \otimes I}\right)=0
$$

because of (4.1) and the positivity of $\langle\cdot, \cdot\rangle_{\phi_{1}}$. Hence, we may assume $u^{(k)} \sim u^{(l)}$ for all $k, l \in\{1, \ldots, n\}$, and apply part (a) to conclude that $\xi=0$.

Finally, as $\langle\cdot, \cdot\rangle_{\phi_{1}}$ is an inner product on $\bigotimes_{i \in I}^{\text {unit }} H_{i}$ and we have both $\bigotimes_{i \in I}^{\text {ct }} H_{i}=$ $\tilde{K} \oplus \bigotimes_{i \in I}^{\text {unit }} H_{i}$ and $\tilde{K} \subseteq K$, we obtain $K \subseteq \tilde{K}$ as well.

(c) Observe that the linear bijection $\Psi:\left(\bigotimes_{i \in I_{1}} H_{i}\right) \otimes\left(\bigotimes_{j \in I_{2}} H_{j}\right) \rightarrow \bigotimes_{i \in I} H_{i}$ as in Remark $2.4(\mathrm{~b})$ restricts to a surjection from $\left(\bigotimes_{i \in I_{1}}^{\text {unit }} H_{i}\right) \otimes\left(\bigotimes_{j \in I_{2}}^{\text {unit }} H_{j}\right)$ to $\bigotimes_{i \in I}^{\text {unit }} H_{i}$. Moreover, for any $u, u^{\prime} \in \Pi_{i \in I_{1}} \mathfrak{S}_{1}\left(H_{i}\right)$ and $v, v^{\prime} \in \Pi_{j \in I_{2}} \mathfrak{S}_{1}\left(H_{j}\right)$, we have $\left(u, u^{\prime}\right) \sim\left(v, v^{\prime}\right)$ as elements in $\Pi_{i \in I} \mathfrak{S}_{1}\left(H_{i}\right)$ if and only if $u \sim u^{\prime}$ and $v \sim v^{\prime}$. Thus, the argument in part (b) tells us that

$$
\begin{aligned}
\left\langle\left(\otimes_{i \in I_{1}} u_{i}\right) \otimes\left(\otimes_{j \in I_{2}} v_{j}\right),\left(\otimes_{i \in I_{1}} u_{i}^{\prime}\right) \otimes\left(\otimes_{j \in I_{2}} v_{j}^{\prime}\right)\right\rangle_{\phi_{1}} & \\
= & \left\langle\otimes_{i \in I_{1}} u_{i}, \otimes_{i \in I_{1}} u_{i}^{\prime}\right\rangle_{\phi_{1}}\left\langle\otimes_{j \in I_{2}} v_{j}, \otimes_{j \in I_{2}} v_{j}^{\prime}\right\rangle_{\phi_{1}} .
\end{aligned}
$$

This shows that $\left.\Psi\right|_{\left(\bigotimes_{i \in I_{1}}^{\text {unit }} H_{i}\right) \otimes\left(\bigotimes_{j \in I_{2}}^{\text {unit }} H_{j}\right)}$ is inner product preserving.

We denote by $\bar{\bigotimes}_{i \in I}^{\mu} H_{i}$ and $\bar{\bigotimes}_{i \in I}^{\phi_{1}} H_{i}$ the completions of $\bigotimes_{i \in I}^{\mu} H_{i}$ and $\bigotimes_{i \in I}^{\text {unit }} H_{i}$, respectively, under the norms induced by $\langle\cdot, \cdot\rangle_{\phi_{1}}$. 
Example 4.3. If $H_{i}=\mathbb{C}(i \in I)$, then the sesquilinear form $\langle\cdot, \cdot\rangle_{\phi_{1}}$ is not positive on the whole space $\otimes_{i \in I} H_{i}$ since $\left\langle\left(\otimes_{i \in I} 1 / 2-\otimes_{i \in I} 2\right),\left(\otimes_{i \in I} 1 / 2-\otimes_{i \in I} 2\right)\right\rangle_{\phi_{1}}=-2$.

Set $\prod_{i \in I}^{\text {eu }} H_{i}:=\left\{x \in \Pi_{i \in I} H_{i}: x_{i} \in \mathfrak{S}_{1}\left(H_{i}\right)\right.$ except for a finite number of $\left.i\right\}$ and let $K$ be an inner product space. A multilinear map $\Phi: \Pi_{i \in I}^{\text {eu }} H_{i} \rightarrow K$ (i.e. $\Phi$ is coordinatewise linear) is said to be componentwise inner product preserving if for any $\mu, \nu \in \Omega_{I ; H}^{\text {unit }}$,

$$
\langle\Phi(x), \Phi(y)\rangle=\delta_{\mu, \nu} \Pi_{i \in I}\left\langle x_{i}, y_{i}\right\rangle \quad\left(x \in \Pi_{i \in I}^{\mu} H_{i} ; y \in \Pi_{i \in I}^{\nu} H_{i}\right),
$$

where $\delta_{\mu, \nu}$ is the Kronecker delta.

Theorem 4.4. (a) $\bar{\bigotimes}_{i \in I}^{\phi_{1}} H_{i} \cong \bar{\bigoplus}_{\mu \in \Omega_{I ; H}^{\text {unit }}}^{\ell^{2}} \bar{\bigotimes}_{i \in I}^{\mu} H_{i}$ canonically as Hilbert spaces.

(b) $\left.\Theta_{H}\right|_{\Pi_{i \in I}^{\mathrm{eu}} H_{i}}: \Pi_{i \in I}^{\mathrm{eu}} H_{i} \rightarrow \bigotimes_{i \in I}^{\mathrm{unit}} H_{i}$ is a componentwise inner product preserving multilinear map. For any inner product space $K$ and any componentwise inner product preserving multilinear map $\Phi: \Pi_{i \in I}^{\mathrm{eu}} H_{i} \rightarrow K$, there is a unique isometry $\tilde{\Phi}: \bigotimes_{i \in I}^{\text {unit }} H_{i} \rightarrow K$ such that $\Phi=\left.\tilde{\Phi} \circ \Theta_{H}\right|_{\Pi_{i \in I}^{\mathrm{eu}} H_{i}}$.

Proof. (a) Clearly, $\bigotimes_{i \in I}^{\text {unit }} H_{i}=\sum_{\mu \in \Omega_{I ; H}^{\text {unit }}} \bigotimes_{i \in I}^{\mu} H_{i}$. Moreover, as in the proof of Lemma $4.2(\mathrm{~b})$, the two subspaces $\bigotimes_{i \in I}^{\mu} H_{i}$ and $\bigotimes_{i \in I}^{\nu} H_{i}$ are orthogonal if $\mu$ and $\nu$ are distinct elements in $\Omega_{I ; H}^{\text {unit }}$. The rest of the argument is standard.

(b) It is easy to see that $\left.\Theta_{H}\right|_{\Pi_{i \in I}^{\text {eu }} H_{i}}$ is componentwise inner product preserving. The uniqueness of $\tilde{\Phi}$ follows from the fact that $\Theta_{H}\left(\Pi_{i \in I}^{\mathrm{eu}} H_{i}\right)$ generates $\bigotimes_{i \in I}^{\text {unit }} H_{i}$. To show the existence of $\tilde{\Phi}$, we first define a multilinear map $\Phi_{0}: \Pi_{i \in I} H_{i} \rightarrow$ $K$ by setting $\Phi_{0}=\Phi$ on $\Pi_{i \in I}^{\mathrm{eu}} H_{i}$ and $\Phi_{0}=0$ on $\Pi_{i \in I} H_{i} \backslash \Pi_{i \in I}^{\mathrm{eu}} H_{i}$. Let $\tilde{\Phi}_{0}$ : $\bigotimes_{i \in I} H_{i} \rightarrow K$ be the induced linear map and set $\tilde{\Phi}:=\left.\tilde{\Phi}_{0}\right|_{\bigotimes_{i \in I}^{\text {unit }} H_{i}}$. Suppose that $u, v \in \Pi_{i \in I} \mathfrak{S}_{1}\left(H_{i}\right), \xi \in \bigotimes_{i \in I}^{u} H_{i}$ and $\eta \in \bigotimes_{i \in I}^{v} H_{i}$. If $u \nsim v$, then $\langle\xi, \eta\rangle_{\phi_{1}}=$ $0=\langle\tilde{\Phi}(\xi), \tilde{\Phi}(\eta)\rangle$. Otherwise, there exist $F \in \mathfrak{F}$ and $\xi_{0}, \eta_{0} \in \bigotimes_{i \in F} H_{i}$ such that $\xi=J_{F}^{u}\left(\xi_{0}\right), \eta=J_{F}^{v}\left(\eta_{0}\right)$ and $u_{i}=v_{i}$ if $i \in I \backslash F$. In this case, $\langle\tilde{\Phi}(\xi), \tilde{\Phi}(\eta)\rangle=$ $\left\langle\xi_{0}, \eta_{0}\right\rangle=\langle\xi, \eta\rangle_{\phi_{1}}$.

Example 4.5. Suppose that $\Phi$ and $\varphi$ are as in Corollary 3.4, and $\left\{\delta_{\mu}\right\}_{\mu \in \Omega_{I ; C}^{\text {unit }}}^{\text {and }}$ is the canonical orthonormal basis for $\ell^{2}\left(\Omega_{I ; \mathbb{C}}^{\text {unit }}\right)$. Note that $\Omega_{I ; \mathbb{C}}^{\text {ut }}=\Omega_{I ; \mathbb{C}}^{\text {unit }}$ and consider the linear bijection $J: \mathbb{C}\left[\Omega_{I ; \mathbb{C}}^{\text {ut }}\right] \rightarrow \mathbb{C}\left[\Omega_{I ; \mathbb{C}}^{\text {unit }}\right]$ given by $J\left(\lambda\left([\alpha]_{\sim}\right)\right):=\delta_{[\alpha]_{\sim}}$ $\left(\alpha \in \Pi_{i \in I} \mathbb{T}\right)$. By Example $2.10(\mathrm{a})$ and Theorem $4.4(\mathrm{a})$, the map $J \circ \Phi$ induces a Hilbert space isomorphism $\hat{\Phi}: \bar{\bigotimes}_{i \in I}^{\phi_{1}} \mathbb{C} \rightarrow \ell^{2}\left(\Omega_{I ; \mathbb{C}}^{\text {unit }}\right)$ such that $\hat{\Phi}\left(\otimes_{i \in I} \beta_{i}\right)=$ $\varphi(\beta) \delta_{[\beta]_{\sim}}\left(\beta \in \Pi_{i \in I} \mathbb{T}\right)$.

We would like to compare $\bar{\bigotimes}_{i \in I}^{\phi_{1}} H_{i}$ with the infinite direct product as defined in [20], when $\left\{H_{i}\right\}_{i \in I}$ is a family of Hilbert spaces. Let us first recall from Definition 3.3.1 in [20] that $x \in \Pi_{i \in I} H_{i}$ is a $C_{0}$-sequence if $\sum_{i \in I}\left|\left\|x_{i}\right\|-1\right|$ converges. As in Definition 3.3.2 in [20], if $x$ and $y$ are $C_{0}$-sequences such that $\sum_{i \in I}\left|\left\langle x_{i}, y_{i}\right\rangle-1\right|$ 
converges, then we write $x \approx y$. Denote by $[x] \approx$ the equivalence class of $x$ under $\approx$, and by $\Gamma_{I ; H}$ the set of all such equivalence classes (see Definition 3.3.3 in [20]).

Let $\prod \otimes_{i \in I} H_{i}$ be the infinite direct product Hilbert space as defined in [20], and let $\prod \otimes_{i \in I} x_{i}$ be the element in $\prod \otimes_{i \in I} H_{i}$ corresponding to a $C_{0}$-sequence $x$ as in Theorem IV of [20]. Notice that if $x \in \Pi_{i \in I}^{\mathrm{eu}} H_{i}$, then $x$ is a $C_{0}$-sequence, and we have a multilinear map

$$
\Upsilon: \prod_{i \in I}^{\mathrm{eu}} H_{i} \longrightarrow \prod \otimes_{i \in I} H_{i}
$$

On the other hand, for any $\mathfrak{C} \in \Gamma_{I ; H}$, we denote by $\prod \otimes_{i \in I}^{\mathfrak{C}} H_{i}$ the closed subspace of $\prod \otimes_{i \in I} H_{i}$ generated by $\left\{\prod \otimes_{i \in I} x_{i}: x \in \mathfrak{C}\right\}$ (see Definition 4.1.1 in [20]).

Proposition 4.6. Let $\left\{H_{i}\right\}_{i \in I}$ be a family of Hilbert spaces.

(a) $[x]_{\sim} \mapsto[x]_{\approx}\left(x \in \Pi_{i \in I} \mathfrak{S}_{1}\left(H_{i}\right)\right)$ gives a well defined surjection $\kappa_{H}: \Omega_{I ; H}^{\text {unit }} \rightarrow$ $\Gamma_{I ; H}$. Moreover, for any $x, y \in \Pi_{i \in I} \mathfrak{S}_{1}\left(H_{i}\right)$, there is a bijection between $\kappa_{H}^{-1}\left([x]_{\approx}\right)$ and $\kappa_{H}^{-1}\left([y]_{\approx}\right)$.

(b) There exists a linear map $\tilde{\Upsilon}: \bigotimes_{i \in I}^{\text {unit }} H_{i} \rightarrow \prod \otimes_{i \in I} H_{i}$ such that $\Upsilon=\tilde{\Upsilon} \circ$ $\left.\Theta_{H}\right|_{\Pi_{i \in I}^{\text {eu }} H_{i}}$ and $\left.\tilde{\Upsilon}\right|_{\bigotimes_{i \in I}^{\mu} H_{i}}$ extends to a Hilbert space isomorphism $\tilde{\Upsilon}^{\mu}: \bar{\bigotimes}_{i \in I}^{\mu} H_{i} \rightarrow$ $\prod \otimes_{i \in I}^{\kappa_{H}(\mu)} H_{i}\left(\mu \in \Omega_{I ; H}^{\text {unit }}\right)$.

Proof. (a) Clearly, if $x \sim z$, then $x \approx z$ and $\kappa_{H}$ is well defined. Lemma 3.3.7 in [20] tells us that $\kappa_{H}$ is surjective. Furthermore, there exists a unitary $u_{i} \in \mathcal{L}\left(H_{i}\right)$ such that $u_{i} x_{i}=y_{i}(i \in I)$, and $\left[u_{i}\right]_{i \in I}$ induces the required bijective correspondence in the second statement.

(b) By the argument of Theorem 4.4 (b), one can construct a linear map $\tilde{\Upsilon}$ such that $\Upsilon=\left.\tilde{\Upsilon} \circ \Theta_{H}\right|_{\Pi_{i \in I}^{\text {eu }} H_{i}}$. By the argument of part (a), we see that $\tilde{\Upsilon}\left(\otimes_{i \in I}^{[u]_{\sim}} H_{i}\right) \subseteq$ $\prod \otimes_{i \in I}^{[u]} H_{i}\left(u \in \prod_{i \in I} \mathfrak{S}_{1}\left(H_{i}\right)\right)$. Furthermore, by Lemma $4.2(\mathrm{a})$, Proposition $4.1(\mathrm{~b})$ and Theorem IV in [20], we see that $\left.\tilde{\Upsilon}\right|_{\bigotimes_{i \in I}^{[u] \sim} H_{i}}$ is an isometry. Finally, $\left.\tilde{\Upsilon}\right|_{\bigotimes_{i \in I}^{[u] \sim H_{i}}}$ has dense range (by Lemma 4.1.2 of [20]).

Notice that $\tilde{\Upsilon}$ is, in general, unbounded but Remark 4.7 (b) below tells us that $\bar{\bigotimes}_{i \in I}^{\phi_{1}} H_{i}$ is a "natural dilation" of $\prod \otimes_{i \in I} H_{i}$. On the other hand, Remark $4.7(\mathrm{~d})$ says that it is possible to construct $\prod \otimes_{i \in I} H_{i}$ in a way similar to $\bar{\bigotimes}_{i \in I}^{\phi_{1}} H_{i}$. Note however, that the construction of $\bar{\bigotimes}_{i \in I}^{\phi_{1}} H_{i}$ is totally algebraic and $\bar{\bigotimes}_{i \in I}^{\phi_{1}} H_{i}$ itself seems to be more natural (see Theorem 4.8 and Example 5.6 below).

Remark 4.7. Suppose that $\left\{H_{i}\right\}_{i \in I}$ is a family of Hilbert spaces.

(a) $\sim$ and $\approx$ are different even in the case when $I=\mathbb{N}$ and $H_{i}=\mathbb{C}(i \in \mathbb{N})$ because one can find $x, y \in \Pi_{i \in \mathbb{N}} \mathbb{T}$ with $x_{i} \neq y_{i}$ for all $i \in \mathbb{N}$ but for which $\sum_{i=1}^{\infty}\left|\left\langle x_{i}, y_{i}\right\rangle-1\right|$ converges. In fact, $\kappa_{H}^{-1}\left([x]_{\approx}\right)$ is an infinite set.

(b) By Lemma 4.1.1 in [20], we have

$$
\prod \otimes_{i \in I} H_{i}=\bar{\bigoplus}_{\mathfrak{C} \in \Gamma_{I ; H}}^{\ell^{2}} \prod \otimes_{i \in I}^{\mathfrak{c}} H_{i} .
$$


Therefore, Theorem 4.4 (a) and Proposition 4.6 tell us that for a fixed $\gamma_{0} \in \Gamma_{I ; H}$, one has a canonical Hilbert space isomorphism

$$
\bar{\bigotimes}_{i \in I}^{\phi_{1}} H_{i} \cong \ell^{2}\left(\kappa_{H}^{-1}\left(\gamma_{0}\right)\right) \bar{\otimes}\left(\prod \otimes_{i \in I} H_{i}\right)
$$

(c) For each $i \in I$, let $K_{i}$ be an inner product space such that $H_{i}$ is the completion of $K_{i}$. Then $\bar{\bigotimes}_{i \in I}^{\phi_{1}} K_{i}$ is, in general, not canonically isomorphic to $\bar{\bigotimes}_{i \in I}^{\phi_{1}} H_{i}$ because $\Omega_{I ; K}^{\text {unit }} \subsetneq \Omega_{I ; H}^{\text {unit }}$ if $K_{i} \subsetneq H_{i}$ for an infinite number of $i \in I$. On the other hand, if $I$ is countable, for any $x \in \Pi_{i \in I} \mathfrak{S}_{1}\left(H_{i}\right)$, there exists $y \in \Pi_{i \in I} \mathfrak{S}_{1}\left(K_{i}\right)$ such that $x \approx y$. This shows that the restriction, $\kappa_{H ; K}$, of $\kappa_{H}$ to $\Omega_{I ; K}^{\text {unit is also a }}$ surjection onto $\Gamma_{I ; H}$. However, we do not know if the cardinality of $\kappa_{H ; K}^{-1}(\mathfrak{C})$ are the same for different $\mathfrak{C} \in \Gamma_{I ; H}$.

(d) If $\phi_{0}$ is as in Example $2.2(\mathrm{~b})$, it is easy to see that

$$
\left\langle\prod \otimes u_{i}, \prod \otimes v_{i}\right\rangle=\phi_{0}\left(\left\langle\otimes_{i \in I} u_{i}, \otimes_{i \in I} v_{i}\right\rangle_{\mathbb{C} \otimes I}\right) \quad\left(u, v \in \Pi_{i \in I}^{\text {unit }} H_{i}\right) .
$$

Thus, the sesquilinear form $\phi_{0}\left(\langle\cdot, \cdot\rangle_{\mathbb{C} \otimes I}\right)$ produces $\prod \otimes H_{i}$. If one wants a selfcontained alternative construction for $\prod \otimes H_{i}$, one needs to establish the positivity of $\phi_{0}\left(\langle\cdot, \cdot\rangle_{\mathbb{C} \otimes I}\right)$, which can be reduced to showing the positivity when all $H_{i}$ are of the same finite dimension.

In the remainder of this section, we show that $\bigotimes_{i \in I}^{\text {unit }} H_{i}$ can be completed into a $C^{*}\left(\Omega_{I ; \mathbb{C}}^{\text {ut }}\right)$-module, which gives many pre-inner products on $\bigotimes_{i \in I}^{\text {unit }} H_{i}$ including $\langle\cdot, \cdot\rangle_{\phi_{1}}$. In the following, we use the convention that the $A$-valued inner product of an inner product $A$-module is $A$-linear in the first variable (where $A$ is a pre- $C^{*}$ algebra). On the other hand, we recall that if $G$ is a group and $\lambda_{g}$ is the canonical image of $g$ in $\mathbb{C}[G]$, the map $\sum_{g \in G} \alpha_{g} \lambda_{g} \mapsto \alpha_{e}\left(\alpha_{g} \in \mathbb{C}\right)$, where $e \in G$ is the identity, extends to a faithful tracial state $\chi_{G}$ on $C^{*}(G)$.

Theorem 4.8. (a) There exists an inner product $\mathbb{C}\left[\Omega_{I ; \mathbb{C}}^{\mathrm{ut}}\right]$-module structure on $\bigotimes_{i \in I}^{\text {unit }} H_{i}$. If $\bar{\bigotimes}_{i \in I}^{\text {mod }} H_{i}$ is the Hilbert $C^{*}\left(\Omega_{I ; \mathbb{C}}^{\text {ut }}\right)$-module given by the completion of this $\mathbb{C}\left[\Omega_{I ; \mathbb{C}}^{\mathrm{ut}}\right]$-module, we have a canonical Hilbert space isomorphism

$$
\bar{\bigotimes}_{i \in I}^{\phi_{1}} H_{i} \cong\left(\bar{\bigotimes}_{i \in I}^{\mathrm{mod}} H_{i}\right) \bar{\otimes}_{\chi_{\Omega_{I ; \mathbb{C}}^{\mathrm{ut}}}} \mathbb{C}
$$

(b) If $G \subseteq \Omega_{I ; \mathbb{C}}^{\text {ut }}$ is a subgroup and $\mathcal{E}_{G}: C^{*}\left(\Omega_{I ; \mathbb{C}}^{\text {ut }}\right) \rightarrow C^{*}(G)$ is the canonical conditional expectation, there is an inner product $\mathbb{C}[G]$-module structure on $\bigotimes_{i \in I}^{\text {unit }} H_{i}$, whose completion coincides with the Hilbert $C^{*}(G)$-module $\left(\bar{\bigotimes}_{i \in I}^{\bmod } H_{i}\right) \bar{\otimes}_{\mathcal{E}_{G}} C^{*}(G)$.

Proof. (a) Clearly, $\bigotimes_{i \in I}^{\text {unit }} H_{i}$ is a $\mathbb{C}_{\mathrm{ut}}^{\otimes I}$-submodule of the $\mathbb{C}^{\otimes I}$-module $\bigotimes_{i \in I} H_{i}$ (see Proposition $2.3(\mathrm{c}))$. Moreover, one has a linear "truncation" $E$ from $\mathbb{C}^{\otimes I}=$ $\left(\bigoplus_{\omega \in \Omega_{I ; \mathbb{C}} \backslash \Omega_{I ; \mathbb{C}}^{\mathrm{ut}}} \otimes_{i \in I}^{\omega} \mathbb{C}\right) \oplus \mathbb{C}_{\mathrm{ut}}^{\otimes I}$ to $\mathbb{C}_{\mathrm{ut}}^{\otimes I}$ sending $(\alpha, \beta)$ to $\beta$. Define

$$
\langle\xi, \eta\rangle_{\mathbb{C}_{\mathrm{ut}}^{\otimes I}}:=E\left(\langle\xi, \eta\rangle_{\mathbb{C} \otimes I}\right) \quad\left(\xi, \eta \in \bigotimes_{i \in I}^{\mathrm{unit}} H_{i}\right)
$$


which is a Hermitian $\mathbb{C}_{\mathrm{ut}}^{\otimes I}$-form because by (3.1), we have

$$
E(a b)=E(a) b \quad \text { and } \quad E\left(a^{*}\right)=E(a)^{*} \quad\left(a \in \mathbb{C}^{\otimes I} ; b \in \mathbb{C}_{\mathrm{ut}}^{\otimes I}\right) .
$$

For any $u, v \in \Pi_{i \in I} \mathfrak{S}_{1}\left(H_{i}\right)$, we write $u \sim_{\mathrm{s}} v$ if there exists $\beta \in \Pi_{i \in I} \mathbb{T}$ such that $u_{i}=\beta_{i} v_{i}$ e.f. Then $\sim_{\mathrm{s}}$ is an equivalence relation on $\Pi_{i \in I} \mathfrak{S}_{1}\left(H_{i}\right)$ satisfying

$$
u \sim_{\mathrm{s}} v \text { if and only if }\left\langle\otimes_{i \in I} u_{i}, \otimes_{i \in I} v_{i}\right\rangle_{\mathbb{C}^{\otimes I}} \in \mathbb{C}_{\mathrm{ut}}^{\otimes I} .
$$

Let $\Phi$ and $\varphi$ be as in Corollary 3.4. Suppose that $\xi=\sum_{k=1}^{n} \alpha_{k} \otimes_{i \in I} u_{i}^{(k)}$ with $\alpha_{1}, \ldots, \alpha_{n} \in \mathbb{C}$ and $u^{(1)}, \ldots, u^{(n)} \in \Pi_{i \in I} \mathfrak{S}_{1}\left(H_{i}\right)$. We first show that $\Phi\left(\langle\xi, \xi\rangle_{\mathbb{C}_{\mathrm{ut}}^{\otimes I}}\right) \in$ $C^{*}\left(\Omega_{I ; \mathbb{C}}^{\mathrm{ut}}\right)_{+}$. As in the proof of Lemma $4.2(\mathrm{~b})$, it suffices to consider the case when $u^{(k)} \sim_{\mathrm{s}} u^{(1)}$ for any $k \in\{1, \ldots, n\}$ (because of relation (4.3)). Let $F \in \mathfrak{F}$ and $\beta^{(1)}, \ldots, \beta^{(n)} \in \Pi_{i \in I} \mathbb{T}$ be such that $u_{i}^{(k)}=\beta_{i}^{(k)} u_{i}^{(1)}(i \in I \backslash F ; k=1, \ldots, n)$. For any $k, l \in\{1, \ldots, n\}$, we have

$$
\Phi\left(\left(\Pi_{i \in F}\left\langle u_{i}^{(k)}, u_{i}^{(l)}\right\rangle_{i}\right)\left(\otimes_{i \in I \backslash F} \beta_{i}^{(k)} \overline{\beta_{i}^{(l)}}\right)\right)=\left\langle\tilde{\varphi}_{F}\left(u^{(k)}\right), \tilde{\varphi}_{F}\left(u^{(l)}\right)\right\rangle_{F},
$$

where $\tilde{\varphi}_{F}\left(u^{(k)}\right):=\left(\varphi\left(\beta^{(k)}\right) \Pi_{i \in F} \beta_{i}^{(k)}\right)^{-1}\left(\otimes_{i \in F} u_{i}^{(k)}\right) \otimes \lambda_{\left[\beta^{(k)}\right]_{\sim}}$ and $\langle\cdot, \cdot\rangle_{F}$ is the canonical $\mathbb{C}\left[\Omega_{I ; \mathbb{C}}^{\text {ut }}\right]$-valued inner product on $\left(\bigotimes_{i \in F} H_{i}\right) \otimes \mathbb{C}\left[\Omega_{I ; \mathbb{C}}^{\text {ut }}\right]$. Therefore,

$$
\Phi\left(\langle\xi, \xi\rangle_{\mathbb{C}_{\mathrm{ut}}^{\otimes I}}\right)=\left\langle\sum_{k=1}^{n} \alpha_{k} \tilde{\varphi}_{F}\left(u^{(k)}\right), \sum_{k=1}^{n} \alpha_{k} \tilde{\varphi}_{F}\left(u^{(k)}\right)\right\rangle_{F} \geq 0 .
$$

Next, we show that $\chi_{\Omega_{I ; \mathbb{C}}^{\text {ut }}} \circ \Phi \circ E=\phi_{1}$. Let $\alpha \in \Pi_{i \in I} \mathbb{C}^{\times}$. If $\alpha \nsim 1$, then $\chi_{\Omega_{I ; \mathbb{C}}^{\text {ut }}} \circ$ $\Phi \circ E\left(\otimes_{i \in I} \alpha_{i}\right)=0\left(\operatorname{as} \Phi\left(E\left(\otimes_{i \in I} \alpha_{i}\right)\right) \notin \mathbb{C} \cdot \lambda_{[1]_{\sim}} \backslash\{0\}\right.$, whether or not $\left.[\alpha]_{\sim} \in \Omega_{I ; \mathbb{C}}^{\text {ut }}\right)$ and we also have $\phi_{1}\left(\otimes_{i \in I} \alpha_{i}\right)=0$. If $\alpha \sim 1$, then $\otimes_{i \in I} \alpha_{i}=\left(\Pi_{i \in I} \alpha_{i}\right)\left(\otimes_{i \in I} 1\right)=$ $\left(\Pi_{i \in I} \alpha_{i}\right) \lambda_{[1]_{\sim}}$, which implies that $\chi_{\Omega_{I ; \mathbb{C}}^{\text {ut }}}\left(\Phi\left(\otimes_{i \in I} \alpha_{i}\right)\right)=\Pi_{i \in I} \alpha_{i}=\phi_{1}\left(\otimes_{i \in I} \alpha_{i}\right)$.

Thus, we have

$$
\chi_{\Omega_{I ; \mathbb{C}}^{\mathrm{ut}}}\left(\Phi\left(\langle\xi, \eta\rangle_{\mathbb{C}_{\mathrm{ut}}^{\otimes I}}\right)\right)=\langle\xi, \eta\rangle_{\phi_{1}} \quad\left(\xi, \eta \in \bigotimes_{i \in I}^{\text {unit }} H_{i}\right) .
$$

As a consequence, if $\Phi\left(\langle\xi, \xi\rangle_{\mathbb{C}_{\mathrm{ut}}^{\otimes I}}\right)=0$, we know from Lemma 4.2 (b) that $\xi=0$. This gives an inner product $\mathbb{C}\left[\Omega_{I ; \mathbb{C}}^{\text {ut }}\right]$-module structure on $\bigotimes_{i \in I}^{\text {unit }} H_{i}$. Furthermore, the required isomorphism $\bar{\bigotimes}_{i \in I}^{\phi_{1}} H_{i} \cong\left(\bar{\bigotimes}_{i \in I}^{\bmod } H_{i}\right) \bar{\otimes}_{\chi_{\Omega_{I}^{\mathrm{ut}} \mathrm{C}}} \mathbb{C}$ also follows from (4.4).

(b) Since $\bigotimes_{i \in I}^{\text {unit }} H_{i}$ is a $\mathbb{C}[G]$-module (we identify $\mathbb{C}[G]$ with $\bigoplus_{\omega \in G} \bigotimes_{i \in I}^{\omega} \mathbb{C}$ under the ${ }^{*}$-isomorphism $\Phi$ of Corollary 3.4), every element in $\left(\otimes_{i \in I}^{\text {unit }} H_{i}\right) \otimes_{\mathbb{C}[G]} \mathbb{C}[G]$ is of the form $\xi \otimes_{\mathbb{C}[G]} 1$ for some $\xi \in \bigotimes_{i \in I}^{\text {unit }} H_{i}$. Moreover, if $\xi, \eta \in \bigotimes_{i \in I}^{\text {unit }} H$, then

$$
\left\langle\xi \otimes_{\mathbb{C}[G]} 1, \eta \otimes_{\mathbb{C}[G]} 1\right\rangle_{\left(\bar{\otimes}_{i \in I}^{\bmod } \mathbb{C}\right) \bar{\otimes}_{\mathcal{E}_{G}} C^{*}(G)}=\mathcal{E}_{G}\left(\Phi\left(\langle\xi, \eta\rangle_{\mathbb{C}_{\mathrm{ut}}^{\otimes I}}\right)\right)=\Phi\left(E_{G}\left(\langle\xi, \eta\rangle_{\mathbb{C} \otimes I}\right)\right),
$$

where $E_{G}$ is the linear "truncation" map from $\mathbb{C}^{\otimes I}$ to $\bigoplus_{\omega \in G} \bigotimes_{i \in I}^{\omega} \mathbb{C}$ defined as in part (a). Therefore, $\Phi\left(E_{G}\left(\langle\cdot, \cdot\rangle_{\mathbb{C} \otimes I}\right)\right)$ is a positive Hermitian $\mathbb{C}[G]$-form on $\bigotimes_{i \in I}^{\text {unit }} H_{i}$. Obviously, $\chi_{\Omega_{I ; \mathbb{C}}^{\text {ut }}}=\chi_{G} \circ \mathcal{E}_{G}$, and by (4.4),

$$
\chi_{G}\left(\Phi\left(E_{G}\left(\langle\xi, \eta\rangle_{\mathbb{C} \otimes I}\right)\right)\right)=\chi_{\Omega_{I ; \mathbb{C}}^{\mathrm{ut}}}\left(\Phi\left(\langle\xi, \eta\rangle_{\mathbb{C}_{\mathrm{ut}}^{\otimes I}}\right)=\langle\xi, \eta\rangle_{\phi_{1}} \quad\left(\xi, \eta \in \bigotimes_{i \in I}^{\mathrm{unit}} H\right) .\right.
$$


This implies that $\Phi\left(E_{G}\left(\langle\cdot, \cdot\rangle_{\mathbb{C} \otimes I}\right)\right)$ is non-degenerate (since $\langle\cdot, \cdot\rangle_{\phi_{1}}$ is non-degenerate by Lemma $4.2(\mathrm{~b}))$. Now, equation (4.5) tells us that the Hilbert $C^{*}(G)$-module $\left(\bar{\bigotimes}_{i \in I}^{\text {mod }} H_{i}\right) \bar{\otimes}_{\mathcal{E}_{G}} C^{*}(G)$ is the completion of $\bigotimes_{i \in I}^{\text {unit }} H_{i}$ under the norm induced by the $\mathbb{C}[G]$-valued inner product $\Phi\left(E_{G}\left(\langle\cdot, \cdot\rangle_{\mathbb{C} \otimes I}\right)\right)$.

Let $\{e\}$ be the trivial subgroup of $\Omega_{I ; \mathbb{C}}^{\mathrm{ut}}$. Since one can identify $E_{\{e\}}$ with $\phi_{1}$ (through the argument of Theorem $4.8(\mathrm{~b})$ ), one has

$$
\bar{\bigotimes}_{i \in I}^{\phi_{1}} H_{i} \cong\left(\bar{\bigotimes}_{i \in I}^{\text {mod }} H_{i}\right) \bar{\otimes}_{\mathcal{E}_{\{e\}}} \mathbb{C}
$$

Remark 4.9. (a) For any subgroup $G \subseteq \Omega_{I ; \mathbb{C}}^{\text {ut }}$ and any faithful state $\varphi$ on $C^{*}(G)$, the Hilbert space

$$
\left(\left(\bar{\bigotimes}_{i \in I}^{\bmod } H_{i}\right) \bar{\otimes}_{\mathcal{E}_{G}} C^{*}(G)\right) \bar{\otimes}_{\varphi} \mathbb{C}
$$

induces an inner product on $\bigotimes_{i \in I}^{\text {unit }} H_{i}$.

(b) If $x \in \Pi_{i \in I}^{0} \mathbb{C}$ (see Example $2.2(\mathrm{~b})$ ), then $\sup _{i \in I}\left|x_{i}\right|<\infty$. This, together with the surjectivity of $\kappa_{\mathbb{C}}$ (see Proposition $4.6(\mathrm{a})$ ), tells us that $\Gamma_{I ; \mathbb{C}}$ is a group under the multiplication: $[x]_{\approx} \cdot[y]_{\approx}:=[x y]_{\approx}\left(\right.$ where $(x y)_{i}:=x_{i} y_{i}$ for any $\left.i \in I\right)$. Moreover, $\kappa_{\mathbb{C}}: \Omega_{I ; \mathbb{C}}^{\text {ut }}=\Omega_{I ; \mathbb{C}}^{\text {unit }} \rightarrow \Gamma_{I ; \mathbb{C}}$ is a group homomorphism, which induces a surjective ${ }^{*}$-homomorphism $\bar{\kappa}_{\mathbb{C}}: C^{*}\left(\Omega_{I ; \mathbb{C}}^{\text {ut }}\right) \rightarrow C^{*}\left(\Gamma_{I ; \mathbb{C}}\right)$.

(c) It is natural to ask whether $\left(\left(\bar{\bigotimes}_{i \in I}^{\bmod } H_{i}\right) \bar{\otimes}_{\bar{\kappa}_{\mathbb{C}}} C^{*}\left(\Gamma_{I ; \mathbb{C}}\right)\right) \bar{\otimes}_{\chi_{\Gamma_{I} ; \mathbb{C}}} \mathbb{C}$ is isomorphic to $\prod \otimes_{i \in I} H_{i}$ canonically. Unfortunately, this is not the case. In fact, for any $x, y \in \Pi_{i \in I}^{\text {unit }} H_{i}$, we write $x \approx_{\mathbb{T}} y$ if there exists $\alpha \in \Pi_{i \in I} \mathbb{T}$ with $\alpha \approx 1$ such that $x_{i}=\alpha_{i} y_{i}$ e.f. It is easy to check that $\approx_{\mathbb{T}}$ is an equivalence relation in general standing strictly between $\sim$ and $\approx$. Moreover, one has

$$
\left\langle\left(\left(\otimes_{i \in I} x_{i}\right) \otimes_{\bar{\kappa}_{\mathbb{C}}} 1\right) \otimes_{\chi_{\Gamma_{I} ; \mathbb{C}}} 1,\left(\left(\otimes_{i \in I} y_{i}\right) \otimes_{\bar{\kappa}_{\mathbb{C}}} 1\right) \otimes_{\chi_{\Gamma_{I} ; \mathbb{C}}} 1\right\rangle=0 \quad \text { whenever } x \not_{\mathbb{T}} y,
$$

while $\left\langle\prod \otimes_{i \in I} x_{i}, \prod \otimes_{i \in I} y_{i}\right\rangle=0$ whenever $x \not z y$. Note however, that if all $H_{i}=\mathbb{C}$, then $\approx_{\mathbb{T}}$ and $\approx$ coincide, and one can show that the two Hilbert spaces $\left(\left(\bar{\bigotimes}_{i \in I}^{\bmod } \mathbb{C}\right) \bar{\otimes}_{\kappa_{\mathbb{C}}} C^{*}\left(\Gamma_{I ; \mathbb{C}}\right)\right) \bar{\otimes}_{\chi_{\Gamma_{I} ; \mathbb{C}}} \mathbb{C}$ and $\prod \otimes_{i \in I} \mathbb{C}$ coincide canonically.

Example 4.10. (a) It is clear that $\bar{\bigotimes}_{i \in I}^{\bmod } \mathbb{C}=C^{*}\left(\Omega_{I ; \mathbb{C}}^{\text {ut }}\right)$. For any state $\varphi$ on $C^{*}\left(\Omega_{I ; \mathbb{C}}^{\text {ut }}\right)$, the Hilbert space $\left(\bar{\bigotimes}_{i \in I}^{\bmod } \mathbb{C}\right) \bar{\otimes}_{\varphi} \mathbb{C}$ is the GNS construction of $\varphi$.

(b) If $G$ is a subgroup of $\Omega_{I ; \mathbb{C}}^{\text {ut }}$, we have

$$
\left(\bar{\bigotimes}_{i \in I}^{\bmod } \mathbb{C}\right) \bar{\otimes}_{\mathcal{E}_{G}} C^{*}(G) \cong \ell^{2}\left(\Omega_{I ; \mathbb{C}}^{\mathrm{ut}} / G\right) \bar{\otimes} C^{*}(G)
$$

In fact, let $q: \Omega_{I ; \mathbb{C}}^{\text {ut }} \rightarrow \Omega_{I ; \mathbb{C}}^{\text {ut }} / G$ be the quotient map and $\sigma: \Omega_{I ; \mathbb{C}}^{\text {ut }} / G \rightarrow \Omega_{I ; \mathbb{C}}^{\text {ut }}$ be a cross section. One has a bijection from $\Omega_{I ; \mathbb{C}}^{\text {ut }}$ to $\left(\Omega_{I ; \mathbb{C}}^{\text {ut }} / G\right) \times G$ sending $\omega$ to $\left(q(\omega), \sigma\left(q(\omega)^{-1}\right) \omega\right)$. 
This gives a bijective linear map $\Delta: \mathbb{C}\left[\Omega_{I ; \mathbb{C}}^{\mathrm{ut}}\right] \rightarrow \bigoplus_{\Omega_{I ; \mathbb{C}}^{\mathrm{ut}} / G} \mathbb{C}[G]$ such that for any $\omega \in \Omega_{I ; \mathbb{C}}^{\mathrm{ut}}$ and $\varepsilon \in \Omega_{I ; \mathbb{C}}^{\mathrm{ut}} / G$,

$$
\Delta\left(\lambda_{\omega}\right)_{\varepsilon}:= \begin{cases}\lambda_{\sigma\left(\varepsilon^{-1}\right) \omega} & \text { if } q(\omega)=\varepsilon \\ 0 & \text { otherwise. }\end{cases}
$$

Let $\Phi: \bigotimes_{i \in I}^{\text {unit }} \mathbb{C}=\mathbb{C}_{\mathrm{ut}}^{\otimes I} \rightarrow \mathbb{C}\left[\Omega_{I ; \mathbb{C}}^{\mathrm{ut}}\right]$ and $\varphi: \Pi_{i \in I} \mathbb{T} \rightarrow \mathbb{T}$ be as in Corollary 3.4. Suppose that $\alpha, \beta \in \Pi_{i \in I} \mathbb{C}^{\times}$. If $\left[\alpha \beta^{-1}\right]_{\sim}$ does not belong to $G$, then we have $E_{G}\left(\left\langle\otimes_{i \in I} \alpha_{i}, \otimes_{i \in I} \beta_{i}\right\rangle_{\mathbb{C} \otimes I}\right)=0$ and

$$
\left\langle\Delta \circ \Phi\left(\otimes_{i \in I} \alpha_{i}\right), \Delta \circ \Phi\left(\otimes_{i \in I} \beta_{i}\right)\right\rangle_{\bigoplus_{\Omega_{I ; \mathbb{C}}^{\ell^{u}} / G}^{2} \mathbb{C}[G]}=0 .
$$

On the other hand, if $\left[\alpha \beta^{-1}\right]_{\sim} \in G$, then

$$
\begin{aligned}
& \left\langle\Delta \circ \Phi\left(\otimes_{i \in I} \alpha_{i}\right), \Delta \circ \Phi\left(\otimes_{i \in I} \beta_{i}\right)\right\rangle_{\bigoplus_{\Omega_{I ; \mathbb{C}}^{\ell^{2}} / G}^{2}} \mathbb{C}[G] \\
& \quad=\varphi\left(\alpha \beta^{-1}\right) \lambda_{\left[\alpha \beta^{-1}\right]_{\sim}}=\Phi\left(\otimes_{i \in I} \alpha_{i} \beta_{i}^{-1}\right)=\Phi\left(E_{G}\left(\left\langle\otimes_{i \in I} \alpha_{i}, \otimes_{i \in I} \beta_{i}\right\rangle_{\mathbb{C} \otimes I}\right)\right) .
\end{aligned}
$$

This shows that $\Delta \circ \Phi$ is an inner product $\mathbb{C}[G]$-module isomorphism from $\bigotimes_{i \in I}^{\text {unit }} \mathbb{C}$ (equipped with the inner product $\mathbb{C}[G]$-module structure as in Theorem $4.8(\mathrm{~b})$ ) onto $\bigoplus_{\Omega_{I ; \mathbb{C}}^{\text {ut }} / G}^{\ell^{2}} \mathbb{C}[G]$.

\section{Tensor products of *-representations of *-algebras}

In this section, $\left\{\left(A_{i}, H_{i}, \Psi_{i}\right)\right\}_{i \in I}$ is a family of unital ${ }^{*}$-representations, in the sense that $A_{i}$ is a unital ${ }^{*}$-algebra, $H_{i}$ is a Hilbert space and $\Psi_{i}: A_{i} \rightarrow \mathcal{L}\left(H_{i}\right)$ is a unital $^{*}$-homomorphism $(i \in I)$.

Suppose that $\Psi_{0}:=\tilde{\bigotimes}_{i \in I} \Psi_{i}: \bigotimes_{i \in I} A_{i} \rightarrow L\left(\bigotimes_{i \in I} H_{i}\right)$ is the map as in Proposition $2.3(\mathrm{c})$. It is easy to check that

$$
\left\langle\Psi_{0}(a) \xi, \eta\right\rangle_{\mathbb{C} \otimes I}=\left\langle\xi, \Psi_{0}\left(a^{*}\right) \eta\right\rangle_{\mathbb{C} \otimes I} \quad\left(a \in \bigotimes_{i \in I} A_{i} ; \xi, \eta \in \bigotimes_{i \in I} H_{i}\right) .
$$

Furthermore, one has the following result (which is more or less well known).

Proposition 5.1. For any $\mu \in \Omega_{I ; H}^{\text {unit }}$, the map $\tilde{\otimes}_{i \in I} \Psi_{i}$ induces a unital ${ }^{*}$-representation $\bigotimes_{i \in I}^{\mu} \Psi_{i}: \bigotimes_{i \in I}^{e} A_{i} \rightarrow \mathcal{L}\left(\bigotimes_{i \in I}^{\mu} H_{i}\right)$. If all the $\Psi_{i}$ are injective, then so is $\bigotimes_{i \in I}^{\mu} \Psi_{i}$.

Consequently, one has a unital *-representation of $\bigotimes_{i \in I}^{e} A_{i}$ on the Hilbert space $\bar{\bigotimes}_{i \in I}^{\phi_{1}} H_{i}$. However, it seems impossible to extend it to a unital *-representation of $\bigotimes_{i \in I} A_{i}$ on $\bar{\bigotimes}_{i \in I}^{\phi_{1}} H_{i}$. The biggest ${ }^{*}$-subalgebra $\bigotimes_{i \in I} A_{i}$ that we can think of, for which such an extension is possible, is the subalgebra $\bigotimes_{i \in I}^{\mathrm{ut}} A_{i}$. Example $5.6(\mathrm{a})$ also tells us that it is probably the right subalgebra to consider. 
Let us digress a little bit and give another ${ }^{*}$-representation of $\bigotimes_{i \in I}^{\mathrm{ut}} A_{i}$, which is a direct consequence of Proposition 5.1, Theorem 3.2 (a) and Theorem 4.1 in [5] (it is not hard to verify that the representation as given in Theorem 4.1 of [5] is injective when $\bigotimes_{i \in I}^{\mu} \Psi_{i}$ is injective). Note however, that such a ${ }^{*}$-representation is not canonical since it depends on the choice of a cross section $c: \Omega_{I ; A}^{\text {ut }} \rightarrow \prod_{i \in I} U_{A_{i}}$ (see Remark $3.3(\mathrm{a})$ ).

Corollary 5.2. Suppose that the $\Psi_{i}$ are injective. For any $\mu \in \Omega_{I ; H}^{\text {unit }}$, the injection $\bigotimes_{i \in I}^{\mu} \Psi_{i}$ induces an injective unital ${ }^{*}$-representation of $\bigotimes_{i \in I}^{\mathrm{ut}} A_{i}$ on $\left(\bar{\bigotimes}_{i \in I}^{\mu} H_{i}\right) \otimes$ $\ell^{2}\left(\Omega_{I ; A}^{\text {ut }}\right)$.

Let us now return to the discussion of the tensor product type representation of $\bigotimes_{i \in I}^{\mathrm{ut}} A_{i}$. Observe that $\left\{\Psi_{i}\right\}_{i \in I}$ induces a canonical action $\alpha^{\Psi}: \Omega_{I ; A}^{\mathrm{ut}} \times \Omega_{I ; H}^{\mathrm{unit}} \rightarrow$ $\Omega_{I ; H}^{\mathrm{unit}}$. For simplicity, we will denote $\alpha_{\omega}^{\Psi}(\mu)$ by $\omega \cdot \mu\left(\omega \in \Omega_{I ; A}^{\mathrm{ut}} ; \mu \in \Omega_{I ; H}^{\mathrm{unit}}\right)$.

Theorem 5.3. (a) The map $\tilde{\bigotimes}_{i \in I} \Psi_{i}$ induces a unital ${ }^{*}$-representation $\bigotimes_{i \in I}^{\phi_{1}} \Psi_{i}$ : $\bigotimes_{i \in I}^{\mathrm{ut}} A_{i} \rightarrow \mathcal{L}\left(\bar{\bigotimes}_{i \in I}^{\phi_{1}} H_{i}\right)$.

(b) $\left(\bar{\bigotimes}_{i \in I}^{\phi_{1}} H_{i},\left.\left(\bigotimes_{i \in I}^{\phi_{1}} \Psi_{i}\right)\right|_{\bigotimes_{i \in I}^{e} A_{i}}\right)=\bigoplus_{\mu \in \Omega_{I ; H}^{\mathrm{unit}}}\left(\bar{\bigotimes}_{i \in I}^{\mu} H_{i}, \bigotimes_{i \in I}^{\mu} \Psi_{i}\right)$.

(c) If all $\Psi_{i}$ are injective, then so is $\bigotimes_{i \in I}^{\phi_{1}} \Psi_{i}$.

Proof. (a) Set $\Psi_{0}:=\tilde{\bigotimes}_{i \in I} \Psi_{i}$. For any $\mu \in \Omega_{I ; H}^{\text {unit }}, \omega \in \Omega_{I ; A}^{\text {ut }}$ and $a \in \prod_{i \in I}^{\omega} A_{i}$, it is clear that

$$
\Psi_{0}\left(\otimes_{i \in I} a_{i}\right)\left(\bigotimes_{i \in I}^{\mu} H_{i}\right) \subseteq \bigotimes_{i \in I}^{\omega \cdot \mu} H_{i}
$$

Suppose that $u \in \omega$ and $F \in \mathfrak{F}$ are such that $a_{i}=u_{i}$ for $i \in I \backslash F$. If $\xi=J_{F^{\prime}}^{x}\left(\xi_{0}\right)$ where $x \in \mu, F^{\prime} \in \mathfrak{F}$ with $F \subseteq F^{\prime}$ and $\xi_{0} \in \bigotimes_{i \in F^{\prime}} H_{i}$, then

$$
\begin{aligned}
\left\langle\Psi_{0}\left(\otimes_{i \in I} a_{i}\right) \xi, \Psi_{0}\right. & \left.\left(\otimes_{i \in I} a_{i}\right) \xi\right\rangle_{\mathbb{C} \otimes I}= \\
& \left\langle\left(\bigotimes_{i \in F} \Psi_{i}\left(a_{i}\right) \otimes \mathrm{id}\right) \xi_{0},\left(\bigotimes_{i \in F} \Psi_{i}\left(a_{i}\right) \otimes \mathrm{id}\right) \xi_{0}\right\rangle\left(\otimes_{i \in I} 1\right) .
\end{aligned}
$$

This implies that $\Psi_{0}\left(\otimes_{i \in I} a_{i}\right)$ is bounded on $\left(\bigotimes_{i \in I}^{\text {unit }} H_{i},\langle\cdot, \cdot\rangle_{\phi_{1}}\right)$ (see Theorem 4.4 (a) and Proposition $4.1(\mathrm{~b}))$ and produces a unital homomorphism $\bigotimes_{i \in I}^{\phi_{1}} \Psi_{i}: \bigotimes_{i \in I}^{\text {ut }} A_{i}$ $\rightarrow \mathcal{L}\left(\bar{\bigotimes}_{i \in I}^{\phi_{1}} H_{i}\right)$. Now, relation (5.1) tells us that $\bigotimes_{i \in I}^{\phi_{1}} \Psi_{i}$ preserves the involution.

(b) This part follows directly from the argument of part (a).

(c) Set $\Psi:=\bigotimes_{i \in I}^{\phi_{1}} \Psi_{i}$. Suppose that $v^{(1)}, \ldots, v^{(n)} \in \Pi_{i \in I} U_{A_{i}}$ are mutually inequivalent elements, $F \in \mathfrak{F}, b^{(1)}, \ldots, b^{(n)} \in \bigotimes_{i \in F} A_{i}$ and $a^{(k)}:=J_{F}^{v^{(k)}}\left(b^{(k)}\right)$ $(k=1, \ldots, n)$ are such that

$$
\Psi\left(\sum_{k=1}^{n} a^{(k)}\right)=0
$$

By induction, it suffices to show that $a^{(1)}=0$. 
By replacing $a^{(k)}$ with $\left(v^{(1)}\right)^{-1} a^{(k)}$ if necessary, we may assume that $v_{i}^{(1)}=e_{i}$ $(i \in I)$. If $n=1$, we take an arbitrary $\xi \in \Pi_{i \in I} \mathfrak{S}_{1}\left(H_{i}\right)$. If $n>1$, we claim that there exists $\xi \in \Pi_{i \in I} \mathfrak{S}_{1}\left(H_{i}\right)$ such that

$$
\xi \nsim\left[V_{i}^{(k)} \xi_{i}\right]_{i \in I} \quad(k=2, \ldots, n),
$$

where $V_{i}^{(k)}:=\Psi_{i}\left(v_{i}^{(k)}\right)$. In fact, if $k \in\{2, \ldots, n\}$ and $i \in I^{k}:=\left\{i \in I: v_{i}^{(k)} \neq e_{i}\right\}$ (which is an infinite set), the subset $\mathfrak{S}_{1}\left(H_{i}\right) \cap \operatorname{ker}\left(V_{i}^{(k)}-\operatorname{id}_{H_{i}}\right)$ is nowhere dense in $\mathfrak{S}_{1}\left(H_{i}\right)$ as $\operatorname{ker}\left(V_{i}^{(k)}-\mathrm{id}_{H_{i}}\right)$ is a proper closed subspace of $H_{i}$ (note that $\Psi_{i}$ is injective). For any $i \in I$, we consider $N_{i}:=\left\{k \in\{2, \ldots, n\}: i \in I^{k}\right\}$. By the Baire category theorem, for every $i \in I$, one can choose $\xi_{i} \in \mathfrak{S}_{1}\left(H_{i}\right) \backslash \bigcup_{k \in N_{i}} \operatorname{ker}\left(V_{i}^{(k)}-\right.$ $\left.\operatorname{id}_{H_{i}}\right)$. Now, $\xi:=\left[\xi_{i}\right]_{i \in I}$ will satisfy relation (5.3).

Since $\Psi\left(a^{(1)}\right)\left(\bigotimes_{i \in I}^{\xi} H_{i}\right) \subseteq \bigotimes_{i \in I}^{\xi} H_{i}$ and

$$
\bigotimes_{i \in I}^{\xi} H_{i} \cap \sum_{k=2}^{n} \Psi\left(a^{(k)}\right)\left(\bigotimes_{i \in I}^{\xi} H_{i}\right)=\{0\}
$$

(because of Theorem 2.5 as well as (5.2) and (5.3)), we have $\left.\Psi\left(a^{(1)}\right)\right|_{\bigotimes_{i \in I}^{\xi} H_{i}}=0$. Therefore, part (b) and Proposition 5.1 tells us that $a^{(1)}=0$.

Remark 5.4. (a) By the argument proving Theorem 5.3 (c), if all the $\Psi_{i}$ are injective, then $\alpha^{\Psi}$ is strongly faithful in the sense that for any finite subset $F \subseteq$ $\Omega_{I ; A}^{\mathrm{ut}} \backslash\{e\}$, there exists $\mu \in \Omega_{I ; H}^{\mathrm{unit}}$ with $\omega \cdot \mu \neq \mu(\omega \in F)$.

(b) If $y, z \in \Pi_{i \in I} H_{i}$ are $C_{0}$-sequences and $u, v \in \Pi_{i \in I} U_{A_{i}}$, then

$$
y \approx z \quad \text { if and only if }\left[\Psi_{i}\left(u_{i}\right) y_{i}\right]_{i \in I} \approx\left[\Psi_{i}\left(u_{i}\right) z_{i}\right]_{i \in I}
$$

and $\left[\Psi_{i}\left(u_{i}\right) y_{i}\right]_{i \in I} \approx\left[\Psi_{i}\left(v_{i}\right) y_{i}\right]_{i \in I}$ whenever $u \sim v$. Thus, $\left\{\Psi_{i}\right\}_{i \in I}$ induces an action $\tilde{\alpha}^{\Psi}: \Omega_{I ; A}^{\mathrm{ut}} \times \Gamma_{I ; H} \rightarrow \Gamma_{I ; H}$. Again, we write $\omega \cdot \gamma$ for $\tilde{\alpha}_{\omega}^{\Psi}(\gamma)\left(\omega \in \Omega_{I ; A}^{\mathrm{ut}} ; \gamma \in \Gamma_{I ; A}\right)$. The map $\kappa_{H}$ in Proposition 4.6 (a) is equivariant in the sense that $\kappa_{H} \circ \alpha_{\omega}^{\Psi}=\tilde{\alpha}_{\omega}^{\Psi} \circ \kappa_{H}$ $\left(\omega \in \Omega_{I ; A}^{\mathrm{ut}}\right)$.

(c) If all the $A_{i}$ are $C^{*}$-algebras and all the $\Psi_{i}$ are irreducible, then $\alpha^{\Psi}$ is transitive.

Corollary 5.5. There exists a unital ${ }^{*}$-representation $\prod \otimes_{i \in I} \Psi_{i}: \bigotimes_{i \in I}^{\mathrm{ut}} A_{i} \rightarrow$ $\mathcal{L}\left(\prod \otimes_{i \in I} H_{i}\right)$ such that for any $\mu \in \Omega_{I ; H}^{\mathrm{unit}}, \omega \in \Omega_{I ; A}^{\mathrm{ut}}$ and $b \in \bigotimes_{i \in I}^{\omega} A_{i}$,

$$
\left(\prod \otimes_{i \in I} \Psi_{i}\right)(b) \circ \tilde{\Upsilon}^{\mu}=\left.\tilde{\Upsilon}^{\omega \cdot \mu} \circ\left(\bigotimes_{i \in I}^{\phi_{1}} \Psi_{i}\right)(b)\right|_{\bar{\bigotimes}_{i \in I}^{\mu} H_{i}},
$$

where $\tilde{\Upsilon}^{\mu}$ is as in Proposition 4.6 (b).

Proof. By Proposition 4.6 (b), there is a bounded linear map

$$
\left(\prod \otimes_{i \in I} \Psi_{i}\right)(b): \prod \otimes_{i \in I}^{\kappa_{H}(\mu)} H_{i} \rightarrow \prod \otimes_{i \in I}^{\omega \cdot \kappa_{H}(\mu)} H_{i}
$$


such that equality (5.5) holds (see also Remark 5.4(b)). Since we have

$$
\sup _{\mu \in \Omega_{I ; H}^{\mathrm{unit}}}\left\|\left.\left(\bigotimes_{i \in I}^{\phi_{1}} \Psi_{i}\right)(b)\right|_{\bar{\bigotimes}_{i \in I}^{\mu} H_{i}}\right\|<\infty
$$

(because of Theorem $5.3(\mathrm{a})$ ), we know from Proposition 4.6 (a) and Lemma 4.1.1 in $[20]$ that $\left(\prod \otimes_{i \in I} \Psi_{i}\right)(b)$ induces an element in $\mathcal{L}\left(\prod \otimes_{i \in I} H_{i}\right)$, which clearly gives a *-representation.

It is natural to ask if $\prod \otimes_{i \in I} \Psi_{i}$ is injective if all the $\Psi_{i}$ are. However, $\prod \otimes_{i \in I} \Psi_{i}$ is never injective as can be seen from Example 5.6 (b) and the discussion following it.

Example 5.6. For any $i \in I$, let $A_{i}=\mathbb{C}=H_{i}$ and let $\iota_{i}: A_{i} \rightarrow \mathcal{L}\left(H_{i}\right)$ be the canonical map. Suppose that $\Phi, \varphi$ and $\hat{\Phi}$ are as in Example 4.5.

(a) Let $\Lambda: \mathbb{C}\left[\Omega_{I ; \mathbb{C}}^{\text {ut }}\right] \rightarrow \mathcal{L}\left(\ell^{2}\left(\Omega_{I ; \mathbb{C}}^{\text {ut }}\right)\right)$ be the left regular representation. For every $\alpha, \beta \in \Pi_{i \in I} \mathbb{T}$, one has

$$
\begin{aligned}
\left(\hat{\Phi}^{*} \circ \Lambda\left(\lambda_{[\alpha]_{\sim}}\right) \circ \hat{\Phi}\right)\left(\otimes_{i \in I} \beta_{i}\right) & =\varphi\left(\alpha^{-1}\right) \otimes_{i \in I} \alpha_{i} \beta_{i} \\
& =\left(\bigotimes_{i \in I}^{\phi_{1}} \iota_{i}\right)\left(\Phi^{-1}\left(\lambda_{[\alpha]_{\sim}}\right)\right)\left(\otimes_{i \in I} \beta_{i}\right) .
\end{aligned}
$$

Consequently, $\bigotimes_{i \in I}^{\phi_{1}} \iota_{i}$ can be identified with $\Lambda$ (under $\Phi$ and $\hat{\Phi}$ ).

(b) Let $\alpha \in \Pi_{i \in I} \mathbb{T}$ be such that $\alpha \nsim 1$ but $\alpha \approx 1$ with $\Pi_{i \in I} \alpha_{i}=1$. If $\beta \in \Pi_{i \in I} \mathbb{C}$ is a $C_{0}$-sequence with $\left\|\prod \otimes_{i \in I} \beta_{i}\right\|=1$, one has $\left\|\prod \otimes_{i \in I} \alpha_{i} \beta_{i}\right\|=1$ and

$$
\left\langle\prod \otimes_{i \in I} \alpha_{i} \beta_{i}, \prod \otimes_{i \in I} \beta_{i}\right\rangle=1
$$

which imply that $\prod \otimes_{i \in I} \alpha_{i} \beta_{i}=\prod \otimes_{i \in I} \beta_{i}$. Therefore, $\left(\prod \otimes_{i \in I} \iota_{i}\right)\left(\otimes_{i \in I} \alpha_{i}\right)=$ id but $\otimes_{i \in I} \alpha_{i} \neq \otimes_{i \in I}$ 1. Consequently, $\prod \otimes_{i \in I} \iota_{i}$ is not injective (actually, $\left(\prod \otimes_{i \in I} \iota_{i}\right) \circ \Phi^{-1}$ is not injective as a group representation of $\left.\Omega_{I ; \mathbb{C}}^{\mathrm{ut}}\right)$.

In general, even $\left.\left(\prod \otimes_{i \in I} \Psi_{i}\right)\right|_{\bigotimes_{i \in I}^{u t} \mathbb{C} e_{i}}$ is not injective. In fact, suppose that $\alpha$ is as above. For any $C_{0}$-sequence $\xi \in \Pi_{i \in I} H_{i}$, with $\left\|\prod \otimes_{i \in I} \xi_{i}\right\|=1$, the same argument as Example $5.6(\mathrm{~b})$ tells us that $\prod \otimes_{i \in I} \alpha_{i} \xi_{i}=\prod \otimes_{i \in I} \xi_{i}$. Thus,

$$
\left(\prod \otimes_{i \in I} \Psi_{i}\right)\left(\otimes_{i \in I} e_{i}-\otimes_{i \in I} \alpha_{i} e_{i}\right)=0
$$

On the other hand, by Theorem 5.3 and Corollary 5.5, there exist canonical *-homomorphisms

$$
J^{\phi_{1}}: \bigotimes_{i \in I}^{\mathrm{ut}} \mathcal{L}\left(H_{i}\right) \rightarrow \mathcal{L}\left(\bigotimes_{i \in I}^{\phi_{1}} H_{i}\right) \text { and } J^{\Pi}: \bigotimes_{i \in I}^{\mathrm{ut}} \mathcal{L}\left(H_{i}\right) \rightarrow \mathcal{L}\left(\prod \otimes_{i \in I} H_{i}\right)
$$

Notice that $J^{\phi_{1}}$ is injective but $J^{\Pi}$ is never injective. 
Corollary 5.7. Let $\pi_{i}: G_{i} \rightarrow U_{\mathcal{L}\left(H_{i}\right)}$ be a unitary representation of a group $G_{i}$, for each $i \in I$.

(a) There exist canonical unitary representations $\bigotimes_{i \in I}^{\phi_{1}} \pi_{i}$ and $\prod \otimes_{i \in I} \pi_{i}$ of $\Pi_{i \in I} G_{i}$ on $\bar{\bigotimes}_{i \in I}^{\phi_{1}} H_{i}$ and $\prod \otimes_{i \in I} H_{i}$ respectively.

(b) If the induced ${ }^{*}$-representation $\hat{\pi}_{i}: \mathbb{C}\left[G_{i}\right] \rightarrow \mathcal{L}\left(H_{i}\right)$ is injective for all $i \in I$, the induced ${ }^{*}$-representation $\widehat{\bigotimes_{i \in I}^{\phi_{1}} \pi_{i}}$ of $\mathbb{C}\left[\Pi_{i \in I} G_{i}\right]$ is also injective.

Proof. (a) Let $\bigotimes_{i \in I}^{\mathrm{ut}} \pi_{i}:=\Theta_{\mathcal{L}(H)} \circ \prod_{i \in I} \pi_{i}: \Pi_{i \in I} G_{i} \rightarrow \bigotimes_{i \in I}^{\mathrm{ut}} \mathcal{L}\left(H_{i}\right)$. Then

$$
\bigotimes_{i \in I}^{\phi_{1}} \pi_{i}:=J^{\phi_{1}} \circ \bigotimes_{i \in I}^{\mathrm{ut}} \pi_{i} \text { and } \prod \otimes_{i \in I} \pi_{i}:=J^{\Pi} \circ \bigotimes_{i \in I}^{\mathrm{ut}} \pi_{i}
$$

are the required representations.

(b) By Theorem $5.3(\mathrm{c}), \bigotimes_{i \in I}^{\phi_{1}} \hat{\pi}_{i}$ is injective. As $\widehat{\bigotimes_{i \in I}^{\phi_{1}} \pi_{i}}$ is the restriction of $\bigotimes_{i \in I}^{\phi_{1}} \hat{\pi}_{i}$ on $\mathbb{C}\left[\Pi_{i \in I} G_{i}\right]$ (see Example $3.1(\mathrm{a})$ ), it is also injective.

Corollary 5.8. $\prod \otimes_{i \in I} \Psi_{i}$ is never irreducible, and neither is $\bigotimes_{i \in I}^{\phi_{1}} \Psi_{i}$.

Proof. Let $\tau_{i}: \mathbb{C} \rightarrow A_{i}$ be the canonical unital map and set $\breve{\Psi}_{i}:=\Psi_{i} \circ \tau_{i}(i \in I)$. Suppose that $\alpha, \beta \in \Pi_{i \in I} \mathbb{T}$ with $\alpha \not \approx \beta$ and $\xi \in \Pi_{i \in I}^{\text {unit }} H_{i}$. Then $\left[\alpha_{i} \xi_{i}\right]_{i \in I} \not \approx\left[\beta_{i} \xi_{i}\right]_{i \in I}$ and the two unit vectors

$$
\left(\prod \otimes_{i \in I} \check{\Psi}_{i}\right)\left(\otimes_{i \in I} \alpha_{i}\right)\left(\prod \otimes_{i \in I} \xi_{i}\right) \text { and }\left(\prod \otimes_{i \in I} \check{\Psi}_{i}\right)\left(\otimes_{i \in I} \beta_{i}\right)\left(\prod \otimes_{i \in I} \xi_{i}\right)
$$

are orthogonal. Consequently, $\operatorname{dim}\left(\prod \otimes_{i \in I} \check{\Psi}_{i}\right)\left(\mathbb{C}_{\mathrm{ut}}^{\otimes I}\right)>1 . \quad$ As $\left(\prod \otimes_{i \in I} \Psi_{i}\right) \circ$ $\left(\otimes_{i \in I} \tau_{i}\right)=\prod \otimes_{i \in I} \check{\Psi}_{i}$, we have $\left(\prod \otimes_{i \in I} \check{\Psi}_{i}\right)\left(\mathbb{C}_{\mathrm{ut}}^{\otimes I}\right) \subseteq Z\left(\left(\prod \otimes_{i \in I} \Psi_{i}\right)\left(\bigotimes_{i \in I}^{\mathrm{ut}} A_{i}\right)\right)$ and $\prod \otimes_{i \in I} \Psi_{i}$ is not irreducible. A similar but easier argument also shows that $\bigotimes_{i \in I}^{\phi_{1}} \Psi_{i}$ is not irreducible.

For any $C^{*}$-algebra $A$, we denote by $S(A)$ and $\left(H_{\rho}, \pi_{\omega}, \xi_{\omega}\right)$ the state space of $A$ and the GNS construction of $\omega \in S(A)$, respectively. We would like to consider a natural injective *-representation of $\bigotimes_{i \in I}^{\mathrm{ut}} A_{i}$ defined in terms of $\left(H_{\omega_{i}}, \pi_{\omega_{i}}\right)$.

If $\rho \in \prod_{i \in I} S\left(A_{i}\right)$ and $\check{\rho}$ is defined as

$$
\check{\rho}(a):=\left\langle\left(\bigotimes_{i \in I}^{\phi_{0}} \pi_{\rho_{i}}\right)(a)\left(\otimes_{i \in I} \xi_{\rho_{i}}\right),\left(\otimes_{i \in I} \xi_{\rho_{i}}\right)\right\rangle \quad\left(a \in \bigotimes_{i \in I}^{\mathrm{ut}} A_{i}\right),
$$

then the closure of $\left(\bigotimes_{i \in I}^{\phi_{1}} \pi_{\rho_{i}}\right)\left(\bigotimes_{i \in I}^{\mathrm{ut}} A_{i}\right)\left(\otimes_{i \in I} \xi_{\rho_{i}}\right)$ will coincide with

$$
H_{\check{\rho}}:=\bar{\bigoplus}_{\omega \in \Omega_{I ; A}^{\mathrm{ut}}} \bar{\bigotimes}_{i \in I}^{\omega \cdot\left[\xi_{\rho}\right] \sim} H_{\rho_{i}} \subseteq \bar{\bigotimes}_{i \in I}^{\phi_{1}} H_{\rho_{i}} .
$$

We set $\pi_{\check{\rho}}(a):=\left.\left(\bigotimes_{i \in I}^{\phi_{1}} \pi_{\rho_{i}}\right)(a)\right|_{H_{\tilde{\rho}}}$. Notice that if all the $\rho_{i}$ are pure states, then $H_{\check{\rho}}=\bar{\bigotimes}_{i \in I}^{\phi_{1}} H_{\rho_{i}}($ see Remark $5.4(\mathrm{c}))$. 
Corollary 5.9. Let $A_{i}$ be a $C^{*}$-algebra $(i \in I)$. The ${ }^{*}$-representation $\Psi_{A}:=$ $\bigoplus_{\rho \in \Pi_{i \in I} S\left(A_{i}\right)}\left(H_{\check{\rho}}, \pi_{\check{\rho}}\right)$ is injective. Consequently, the ${ }^{*}$-representation

$$
\Phi_{A}:=\bigoplus_{\rho \in \Pi_{i \in I} S\left(A_{i}\right)}\left(\bar{\bigotimes}_{i \in I}^{\phi_{1}} H_{\rho_{i}}, \bigotimes_{i \in I}^{\phi_{1}} \pi_{\rho_{i}}\right)
$$

is also injective.

Proof. Suppose that $\left(H_{i}, \Psi_{i}\right)$ is a universal ${ }^{*}$-representation of $A_{i}(i \in I)$. Let $F$, $u^{(1)}, \ldots, u^{(n)}, b^{(1)}, \ldots, b^{(n)}$, and $a^{(1)}, \ldots, a^{(n)}$ be as in the proof of Theorem $5.3(\mathrm{c})$ with $\Psi_{A}\left(\sum_{k=1}^{n} a^{(k)}\right)=0$. Again, it suffices to show that $a^{(1)}=0$, and we may assume that $u_{i}^{(1)}=e_{i}(i \in I)$. If $n=1$, we take any $x \in \Pi_{i \in I} \mathfrak{S}_{1}\left(H_{i}\right)$. If $n>1$, we take an element $x \in \Pi_{i \in I} \mathfrak{S}_{1}\left(H_{i}\right)$ satisfying

$$
x \nsim\left[\Psi_{i}\left(u_{i}^{(k)}\right) x_{i}\right]_{i \in I} \quad(k=2, \ldots, n)
$$

(the argument of Theorem $5.3(\mathrm{c})$ ensures its existence). Let us set $\rho_{i}(a):=$ $\left\langle\Psi_{i}(a) x_{i}, x_{i}\right\rangle$ when $i \in I \backslash F$, and pick any $\rho_{i} \in S\left(A_{i}\right)$ when $i \in F$. For every $i \in I \backslash F$, one may regard $\left(H_{\rho_{i}}, \pi_{\rho_{i}}\right)$ as a subrepresentation of $\left(H_{i}, \Psi_{i}\right)$ such that $\xi_{\rho_{i}} \in H_{\rho_{i}}$ is identified with $x_{i} \in H_{i}$. Then $x$ can be considered as an element in $H_{\check{\rho}}$. Since $x \nsim\left[\pi_{\rho_{i}}\left(u_{i}^{(k)}\right) x_{i}\right]_{i \in I}$ for all $2 \leq k \leq n$, the argument of Theorem 5.3 (c) tells us that

$$
\left(\bigotimes_{i \in I}^{[x]} \pi_{\rho_{i}}\right)\left(a^{(1)}\right) \eta=0 \quad\left(\eta \in \bigotimes_{i \in I}^{x} H_{\rho_{i}}\right) .
$$

Consequently, $\left(\bigotimes_{i \in F} \pi_{\rho_{i}}\right)\left(b^{(1)}\right)=0$ and $b^{(1)}=0$ (as $\rho_{i}$ is arbitrary when $\left.i \in F\right)$. The second statement follows readily from the first one.

Notice also that $\left(\bar{\bigotimes}_{i \in I}^{\phi_{1}} H_{\rho_{i}}, \bigotimes_{i \in I}^{\phi_{1}} \pi_{\rho_{i}}\right)$ is in general not a cyclic representation, and $\left(H_{\check{\rho}}, \pi_{\check{\rho}}\right)$ can be regarded as a cyclic analogue of it.

We end this paper with the following result concerning tensor products of Hilbert algebras.

Corollary 5.10. Let $\left\{A_{i}\right\}_{i \in I}$ be a family of unital Hilbert algebras (see, e.g., Definition VI.1.1 in [18]) such that $\left\|e_{i}\right\|=1(i \in I)$. Then $A:=\bigotimes_{i \in I}^{\mathrm{ut}} A_{i}$ is also a unital Hilbert algebra with $\left\|\otimes_{i \in I} e_{i}\right\|=1$.

Proof. Note that since $\left\|e_{i}\right\|=1$, one has $\left\|u_{i}\right\|=1$ for any $u_{i} \in U_{A_{i}}$. Thus, we have $\bigotimes_{i \in I}^{\text {ut }} A_{i} \subseteq \bigotimes_{i \in I}^{\text {unit }} A_{i}$, which gives an inner product $\langle\cdot, \cdot\rangle_{A}$ on $A$. Observe that $\bigotimes_{i \in I}^{\omega} A_{i}$ is orthogonal to $\bigotimes_{i \in I}^{\omega^{\prime}} A_{i}$ (in terms of $\langle\cdot, \cdot\rangle_{A}$ ) whenever $\omega$ and $\omega^{\prime}$ are distinct elements in $\Omega_{I ; A}^{\mathrm{ut}}$. Thus, in order to show that the involution of $A$ is an isometry, it suffices to check that $\left\|x^{*}\right\|=\|x\|$ whenever $x \in \bigotimes_{i \in I}^{\omega} A_{i}$ and $\omega \in \Omega_{I ; A}^{\mathrm{ut}}$. In fact, for any $u \in \Pi_{i \in I} U_{A_{i}}, F \in \mathfrak{F}$ and $a \in \bigotimes_{i \in F} A_{i}$, we have

$$
\left\|J_{F}^{u}(a)^{*}\right\|=\left\|J_{F}^{u^{*}}\left(a^{*}\right)\right\|=\left\|a^{*}\right\|=\|a\|=\left\|J_{F}^{u}(a)\right\|,
$$


because the involution of $\bigotimes_{i \in F} A_{i}$ is an isometry. Let $H_{i}$ be the completion of $A_{i}$ (with respect to the inner product) and let $\Psi_{i}: A_{i} \rightarrow \mathcal{L}\left(H_{i}\right)$ be the canonical unital ${ }^{*}$-representation $(i \in I)$. Since

$$
\bigotimes_{i \in I}^{\phi_{1}} \Psi_{i}(a) b=a b \quad(a, b \in A),
$$

Theorem 5.3 (a) tells us that for each $x \in A$, one has $\langle x y, z\rangle_{A}=\left\langle y, x^{*} z\right\rangle_{A}(y, z \in A)$ and $\sup _{\|y\| \leq 1}\|x y\|<\infty$. Finally, as $A$ is unital, we see that $A$ is a Hilbert algebra (with $\left\|\otimes_{i \in I} e_{i}\right\|=1$ ).

Consequently, if all the $A_{i}$ are weakly dense unital ${ }^{*}$-subalgebras of finite von Neumann algebras, then so is $\bigotimes_{i \in I}^{\text {ut }} A_{i}$.

\section{References}

[1] Araki, H. and Nakagami, Y.: A remark on an infinite tensor product of von Neumann algebras. Publ. Res. Inst. Math. Sci. 8 (1972), 363-374.

[2] BÉdos, E. And Conti, R.: On infinite tensor products of projective unitary representations. Rocky Mountain J. Math. 34 (2004), 467-493.

[3] Bergmann, W. And Conti, R.: On infinite tensor products of Hilbert $C^{*}$-bimodules. In Operator algebras and mathematical physics (Constanta 2001) 23-34. Theta, Bucharest, 2003.

[4] Blackadar, B.: Infinite tensor products of $C^{*}$-algebras. Pacific J. Math. 77 (1977), no. 2, 313-334.

[5] Busby, R. C. And Smith, H. A.: Representations of twisted group algebras. Trans. Amer. Math. Soc. 149 (1970), 503-537.

[6] Calabi, L.: Sur les extensions des groupes topologiques. Ann. Mat. Pura Appl. (4) 32 (1951), 295-370.

[7] Eilenberg, S. And Maclane, S.: Group extensions and homology. Ann. of Math. (2) 43 (1942), 757-831.

[8] Evans, D. E. And Kawahigashi, Y.: Quantum symmetries on operator algebras. Oxford Mathematical Monographs, Oxford Science Publications, The Clarendon Press, Oxford University Press, New York, 1998.

[9] Floricel, R.: Infinite tensor products of spatial product systems. Infin. Dimens. Anal. Quantum Probab. Relat. Top. 11 (2008), 447-465.

[10] Huo, Y.-P. AND NG, C.-K.: Some algebraic structures related to a quantum system with infinite degrees of freedom. Rep. Math. Phys. 67 (2011), no. 1, 97-107.

[11] Gill, T. L.: Infinite tensor products of Banach spaces. I. J. Funct. Anal. 30 (1978), no. $1,17-35$.

[12] Giordano, T. and Skandalis, G.: On infinite tensor products of factors of type $\mathrm{I}_{2}$. Ergodic Theory Dynam. Systems 5 (1985), 565-586.

[13] Guichardet, A.: Tensor products of $C^{*}$-algebra. II: Infinite tensor products. Lecture Note Series 13, Aarhus Universitet, Matematisk Institut, 1969.

[14] Grundling, H. And Neeb, K.-L.: Infinite tensor products of $C_{0}(\mathbb{R})$ : towards a group algebra for $\mathbb{R}^{(\mathbb{N})}$. To appear in J. Operator Theory. 
[15] Power, S.: Infinite tensor products of upper triangular matrix algebras. Math. Scand. 65 (1989), 291-307.

[16] Raeburn, I., Sims, A. And Williams, D. P.: Twisted actions and obstructions in group cohomology. In $C^{*}$-algebras (Münster, 1999), 161-181. Springer, Berlin, 2000.

[17] Størmer, E.: On infinite tensor products of von Neumann algebras. Amer. J. Math. 93 (1971), 810-818.

[18] Takesaki, M.: Theory of operator algebras II. Encyclopaedia of Mathematical Sciences 125, Operator Algebras and Non-commutative Geometry 6, Springer-Verlag, Berlin, 2003.

[19] Thiemann, T. and Winkler, O.: Gauge field theory coherent states (GCS): IV. Infinite tensor product and thermodynamical limit. Classical Quantum Gravity 18 (2001), 4997-5053.

[20] von Neumann, J.: On infinite direct products. Compositio Math. 6 (1937), 1-77.

[21] Wagner, B. H.: Infinite tensor products of commutative subspace lattices. Proc. Amer. Math. Soc. 103 (1988), 429-437.

Received May 27, 2011; revised November 2, 2011.

Chi-Keung NG: Chern Institute of Mathematics and LPMC, Nankai University, Tianjin 300071, China.

E-mail: ckng@nankai.edu.cn 